\title{
ISOSPECTRAL POTENTIALS AND CONFORMALLY EQUIVALENT ISOSPECTRAL METRICS ON SPHERES, BALLS AND LIE GROUPS
}

\author{
Carolyn S. Gordon and Dorothee Schueth \\ To the memory of our colleague and friend Robert Brooks \\ (1952-2002), whose influence is felt throughout our work.
}

\begin{abstract}
We construct pairs of conformally equivalent isospectral Riemannian metrics $\varphi_{1} g$ and $\varphi_{2} g$ on spheres $S^{n}$ and balls $B^{n+1}$ for certain dimensions $n$, the smallest of which is $n=7$, and on certain compact simple Lie groups. In the case of Lie groups, the metric $g$ is left-invariant. In the case of spheres and balls, the metric $g$ is not the standard metric but may be chosen arbitrarily close to the standard one. For the same manifolds $(M, g)$ we also show that the functions $\varphi_{1}$ and $\varphi_{2}$ are isospectral potentials for the Schrödinger operator $\hbar^{2} \Delta+\varphi$. To our knowledge, these are the first examples of isospectral potentials and of isospectral conformally equivalent metrics on simply connected closed manifolds.
\end{abstract}

\section{INTRODUCTION}

Let $M$ be a compact Riemannian manifold. Two fundamental questions in spectral geometry are:

- To what extent can one recover the geometry of $M$ from the eigenvalue spectrum of the Laplace-Beltrami operator $\Delta$ acting on smooth functions on $M$ ? (If the manifold has boundary, one may impose either Dirichlet or Neumann boundary conditions on the functions.)

- To what extent does the eigenvalue spectrum of the Schrödinger operator $\hbar^{2} \Delta+\varphi$ determine the potential $\varphi \in C^{\infty}(M)$ ?

Various geometric invariants of Riemannian manifolds such as dimension, volume and total scalar curvature are known to be spectrally determined. The primary methods for identifying spectral invariants is through the study of the heat and wave equations. If two Schrödinger operators $\hbar^{2} \Delta+\varphi$ and $\hbar^{2} \Delta+\psi$ on a fixed

Key words and phrases. Laplace operator, spectrum, Schrödinger operator, conformally equivalent metrics, isospectral potentials.

2000 Mathematics Subject Classification. 58J53, 58J50.

The first author is partially supported by National Science Foundation grant DMS-0072534. The second author is partially supported by SFB 611, Bonn. 
Riemannian manifold $M$ are isospectral, then the heat invariants show that $\varphi$ and $\psi$ have the same average and the same $L^{2}$-norm (see, e.g., [Gi]).

We will say two compact Riemannian manifolds are isospectral if the associated Laplacians have the same eigenvalue spectrum. We will say that two potentials $\varphi_{1}$ and $\varphi_{2}$ are isospectral if the Schrödinger operators $\hbar^{2} \Delta+\varphi_{1}$ and $\hbar^{2} \Delta+\varphi_{2}$ are isospectral for every choice of the Planck's constant $\hbar$. The only way of identifying specific geometric invariants, respectively properties of potentials, which are not spectrally determined is through construction of isospectral metrics or potentials. The primary results of this article are the following constructions:

(A) Isospectral conformally equivalent Riemannian metrics on simply-connected manifolds, including spheres of dimension seven and higher, balls of dimension at least eight, and on the Lie groups $\operatorname{Spin}(2 m+4), m \geq 5$ and $\mathrm{SU}(2 m+3), m \geq 3$. The metrics on the spheres, respectively balls, may be chosen arbitrarily close to the round, respectively flat metrics. The metrics on the Lie groups are conformally equivalent to left-invariant metrics.

(B) Isospectral potentials for the Schrödinger operators associated with (noncanonical) Riemannian metrics on the same underlying simply-connected manifolds as in (A). Again the Riemannian metrics on the balls and spheres may be chosen arbitrarily close to the standard ones, and the Riemannian metrics on the Lie groups are left-invariant.

To our knowledge, these constructions give the first examples of isospectral conformally equivalent metrics and of isospectral potentials on simply-connected closed manifolds.

We briefly review the literature on isospectral metrics and potentials. (We do not give a complete list of all known examples.)

The known methods for constructing isospectral manifolds roughly fall into three classes: "Explicit" computations, representation theoretic or trace formula methods, and the use of torus actions. Among the examples constructed by more or less explicit computations are 16-dimensional tori constructed by J. Milnor [Mi] in 1964 (these are the first known examples of isospectral manifolds), spherical space forms $[\mathrm{Ik} 1,2]$, domains in $\mathbb{R}^{4}[\mathrm{Ur}]$, flat manifolds exhibiting various interesting properties [MR1-3], and the first examples of isospectral manifolds with different local geometry [Sz1] and of pairs of isospectral metrics on spheres [Sz2]. Representation methods were first used to construct isospectral Riemann surfaces [Vi] and continuous isospectral deformations [GW1]. In 1985, T. Sunada [Su] gave an elegant trace formula method (which may also be viewed as a representation theoretic method) for constructing isospectral manifolds with a common finite cover. This method and various generalizations $[\mathrm{Be} 1,2],[\mathrm{DG}],[\mathrm{Pe} 1,2]$ led to a vast literature of isospectral manifolds, including isospectral Riemann surfaces of every genus greater than three $[\mathrm{BT}],[\mathrm{Bu}],[\mathrm{BGG}]$, isospectral plane domains $[\mathrm{GWW}]$, and many other examples. The third method, that of torus actions, was developed in the series 
of papers [Go1,3], [GW2], [GGSWW], [GSz], [Sch1-3]; see also [Ba]. This method generally produces manifolds with different local geometry, including among others the first examples of isospectral simply-connected manifolds (in fact, continuous deformations) [Sch1], isospectral deformations of left-invariant metrics on simplyconnected compact Lie groups [Sch2], and isospectral deformations of metrics on spheres [Go2], [Sch3]. Recently, C. Sutton [St] modified Sunada's method (the second method above) to construct new and very different examples of isospectral simply connected manifolds.

R. Brooks, P. Perry and P. Yang [BPY] gave a modification of Sunada's method which yields isospectral conformally equivalent Riemannian metrics with the same local but different global geometry. (This method is closely related to an earlier modification of Sunada's method by $\mathrm{R}$. Brooks $[\mathrm{Br}]$ which produces isospectral potentials for the Schrödinger operator.) The second author [Sch2] analogously extended the method of torus actions to construct the first examples of isospectral conformally equivalent metrics that do not lift to isometric metrics on the universal covering. These were conformally equivalent to left-invariant metrics on products $G \times T$, where $G$ was a simple compact Lie group $(\operatorname{Spin}(n), \mathrm{SO}(n)$ or $\mathrm{SU}(n)$ for various $n$ ) and $T$ was a torus. These examples, together with the constructions of isospectral metrics on Lie groups and spheres referred to above, motivated our construction $(\mathrm{A})$.

For the Schrödinger operator, there is a vast literature concerning isospectral potentials on the circle and on the interval. See, for example, $[\mathrm{PT}]$. An especially interesting connection exists with the Korteveg de Vries equation. Letting an arbitrary function evolve according to the Korteveg de Vries equation, one obtains a continuous family of potentials $\varphi_{t}$ on the circle such that the operators $\Delta+\varphi_{t}$ are mutually isospectral [GGKM]. In higher dimensions, Brooks' modification of Sunada's method, cited above, has yielded isospectral potentials on negatively curved manifolds $[\mathrm{Br}]$ and isospectral deformations of potentials [BG]. Although it was not mentioned explicitly in those two papers, these potentials are isospectral in the strong sense defined above; i.e., they are isospectral for the Schrödinger operators with arbitrary Planck constant $\hbar$. In contrast to the potentials that we will construct, Brooks' method yields potentials that lift to congruent potentials on the universal covering manifold.

The paper is organized as follows: In Section 1 we present the general principles which will be used for constructing conformally equivalent isospectral metrics and isospectral potentials. In Section 2 we outline a strategy for proving nonisometry. Sections 3 and 4 contain the actual examples: Lie groups in Section 3, balls and spheres in Section 4. In Section 5 we prove nonisometry results for the isospectral metrics and potentials constructed in the previous two sections. The stategy outlined in Section 2 is applied in the case of the ball and sphere examples; a different approach is used in the Lie groups case. Finally, in Section 6 we give a short survey 
of how our technique may be applied in other settings.

It is a pleasure to thank Steven Zelditch for helpful comments.

As we were completing this paper, we learned with shock and sorrow of the untimely passing of our close friend and colleague, Robert Brooks. Brooks brought to inverse spectral geometry ideas from such diverse areas as complex analysis, probability and combinatorics. His generosity and enthusiasm in sharing his insights has profoundly affected our research. He is deeply missed.

\section{$\S 1$ Principles for constructing isospectral potentials AND CONFORMALLY EQUIVALENT ISOSPECTRAL METRICS}

\subsection{Definition.}

(i) The spectrum of a closed Riemannian manifold $(M, g)$ is the spectrum of eigenvalues, counted with multiplicities, of the associated Laplace operator $\Delta_{g}$ acting on functions. The Dirichlet spectrum of a compact Riemannian manifold $(M, g)$ with boundary is the spectrum of eigenvalues corresponding to eigenfunctions which satisfy the Dirichlet boundary condition $\left.f\right|_{\partial M}=0$. The Neumann spectrum of such a manifold is defined analogously with respect to the Neumann boundary condition $N f=0$, where $N$ is the inward-pointing unit normal field on the boundary. Given any potential function $\varphi \in C^{\infty}(M)$ and a Planck constant $\hbar \neq 0$, one analogously defines the (Dirichlet, Neumann) spectrum of the operator $\hbar^{2} \Delta_{g}+\varphi: f \mapsto \hbar^{2} \Delta_{g} f+\varphi f$.

(ii) Two closed Riemannian manifolds are called isospectral if they have the same spectrum (including multiplicities). Two compact Riemannian manifolds with boundary are called Dirichlet isospectral, resp. Neumann isospectral, if they have the same Dirichlet spectrum, resp. the same Neumann spectrum. Isospectrality of two operators $\hbar^{2} \Delta_{g}+\varphi$ and $\hbar^{2} \Delta_{g^{\prime}}+\psi$ is defined analogously. If $(M, g)=\left(M^{\prime}, g^{\prime}\right)$ and if $\hbar^{2} \Delta_{g}+\varphi$ and $\hbar^{2} \Delta_{g}+\psi$ are (Dirichlet, Neumann) isospectral for each $\hbar \neq 0$, then $\varphi$ and $\psi$ are called (Dirichlet, Neumann) isospectral potentials on $(M, g)$.

1.2 Notation. By a torus, we always mean a nontrivial, compact, connected abelian Lie group. If a torus $T$ acts smoothly and effectively by isometries on a compact connected Riemannian manifold $(M, g)$, then we denote by $\hat{M}$ the union of those orbits on which $T$ acts freely. Note that $\hat{M}$ is an open dense submanifold of $M$. The action of $T$ gives $\hat{M}$ the structure of a principal $T$-bundle. By $g_{0}^{T}$ we

denote the unique Riemannian metric on the quotient manifold $\hat{M} / T$ such that the canonical projection $\pi:\left(\hat{M}, g_{0}\right) \rightarrow\left(\hat{M} / T, g_{0}^{T}\right)$ is a Riemannian submersion.

1.3 Theorem. Let $T$ be a torus which acts effectively by isometries on two compact connected Riemannian manifolds $(M, g)$ and $\left(M^{\prime}, g^{\prime}\right)$. Let $\varphi \in C^{\infty}(M)$ and $\psi \in$ $C^{\infty}\left(M^{\prime}\right)$ be two potential functions. For each subtorus $W \subset T$ of codimension one, suppose that there exists a T-equivariant diffeomorphism $F_{W}: M \rightarrow M^{\prime}$ which 
satisfies:

(a) $F_{W}^{*} d v o l_{g^{\prime}}=d v o l_{g}$,

(b) $F_{W}$ induces an isometry $\bar{F}_{W}:\left(\hat{M} / W, g^{W}\right) \rightarrow\left(\hat{M}^{\prime} / W, g^{\prime W}\right)$,

(c) $F_{W}^{*} \psi=\varphi$.

Then for each $\hbar \neq 0$, the operators $\hbar^{2} \Delta_{g}+\varphi$ on $M$ and $\hbar^{2} \Delta_{g^{\prime}}+\psi$ on $M^{\prime}$ are isospectral; if the manifolds have boundary then these operators are Dirichlet and Neumann isospectral.

In particular, if moreover there exists an isometry $\tau:(M, g) \rightarrow\left(M^{\prime}, g^{\prime}\right)$ such that $\tau^{*} \psi \neq \varphi$, then $\varphi$ and $\tau^{*} \psi$ are (Dirichlet and Neumann) isospectral potentials on $(M, g)$.

Note that for $\varphi=\psi=0$, this is just Theorem 1.4 from [Sch3], which in turn was a slightly improved version of Theorem 1.2 from [Go3].

Proof. Let $\mathcal{H}$ denote the Sobolev space $H^{1,2}(M, g)$ in the case of closed $M$ or in the case of Neumann boundary conditions, and let $\mathcal{H}$ denote the closure of $C_{0}^{\infty}(M)$ in $H^{1,2}(M, g)$ in the case of Dirichlet boundary conditions. For any subtorus $W$ of $T$ let $\mathcal{H}_{W} \subset \mathcal{H}$ denote the subspace of $W$-invariant functions. Analogously, we define the function spaces $\mathcal{H}^{\prime}$ and $\mathcal{H}_{W}^{\prime}$ on $M^{\prime}$.

The key point in the proof of Theorem 1.4 from [Sch3] (corresponding to the case $\varphi=\psi=0$ ) was deducing from (a) and (b) that for each $W$ of codimension 1 , the pullback

$$
F_{W}^{*}: \mathcal{H}_{W}^{\prime} \rightarrow \mathcal{H}_{W}
$$

preserves both the $L^{2}$ - and the $H^{1,2}$-norm. Combining these maps according to the Fourier decomposition

$$
\mathcal{H}=\mathcal{H}_{T} \oplus \bigoplus_{W}\left(\mathcal{H}_{W} \ominus \mathcal{H}_{T}\right)
$$

one thus obtained an $L^{2}$-norm preserving Hilbert space isometry $I: \mathcal{H}^{\prime} \rightarrow \mathcal{H}$. The statement (for $\varphi=\psi=0$ ) then followed from the variational characterization of eigenvalues via the Rayleigh quotient.

We need only slightly extend this proof in order to obtain Theorem 1.3: By condition (c), together with (a), the pullbacks $F_{W}^{*}$ now also satisfy $\int_{M} \varphi\left|F_{W}^{*} f\right|^{2} d v o l_{g}$ $=\int_{M^{\prime}} \psi|f|^{2} d v o l_{g^{\prime}}$ for all $f \in \mathcal{H}_{W}^{\prime}$. In particular, for the $L^{2}$-norm preserving isometry $I: \mathcal{H}^{\prime} \rightarrow \mathcal{H}$ defined above, we now have

$$
\mathcal{R}_{\varphi}(\text { If })=\mathcal{R}_{\psi}(f)
$$

for all $f \in \mathcal{H}^{\prime} \backslash\{0\}$, where

$$
\begin{aligned}
\mathcal{R}_{\psi}(f): & =\int_{M^{\prime}}\left(h^{2}\|d f\|_{g^{\prime}}^{2}+\psi|f|^{2}\right) d v o l_{g^{\prime}} / \int_{M^{\prime}}|f|^{2} d v o l_{g^{\prime}} \\
& =\left(\left(h^{2}\|f\|_{H^{1,2}\left(M^{\prime}, g^{\prime}\right)}^{2}+\int_{M^{\prime}} \psi|f|^{2} d v o l_{g^{\prime}}\right) /\|f\|_{L^{2}\left(M^{\prime}, g^{\prime}\right)}^{2}\right)-h^{2} .
\end{aligned}
$$


is the modified Rayleigh quotient occurring in the variational characterization of the eigenvalues of $\hbar^{2} \Delta_{g^{\prime}}+\psi$, and where $\mathcal{R}_{\varphi}$ is defined analogously.

Thus the theorem follows again from the variational characterization of eigenvalues.

1.4 Notation. We fix a torus $T$ with Lie algebra $\mathfrak{z}$. Let $\mathcal{L}$ be the cocompact lattice in $\mathfrak{z}$ such that $\exp : \mathfrak{z} \rightarrow T$ induces an isomorphism from $\mathfrak{z} / \mathcal{L}$ to $T$, and denote by $\mathcal{L}^{*} \subset \mathfrak{z}^{*}$ the dual lattice. We also fix a compact connected Riemannian manifold $\left(M, g_{0}\right)$, with or without boundary, and a smooth effective action of $T$ on $\left(M, g_{0}\right)$ by isometries.

(i) For $Z \in \mathfrak{z}$ we denote by $Z^{*}$ the vector field $\left.x \mapsto \frac{d}{d t}\right|_{t=0} \exp (t Z) x$ on $M$. For each $x \in M$, we let $\mathfrak{z}_{x}:=\left\{Z_{x}^{*} \mid Z \in \mathfrak{z}\right\} \subset T_{x} M$.

(ii) We call a smooth 1 -form on $M$ admissible if it is $T$-invariant and horizontal (i.e., vanishes on the vertical spaces $\mathfrak{z} x$ ).

(iii) For any admissible $\mathfrak{z}$-valued 1 -form $\lambda$ on $M$ we denote by $g_{\lambda}$ the Riemannian metric on $M$ given by

$$
g_{\lambda}(X, Y)=g_{0}\left(X+\lambda(X)^{*}, Y+\lambda(Y)^{*}\right) .
$$

Note that $g_{\lambda}$ is the pullback of $g_{0}$ by a field of unipotent endomorphisms; in particular, $d v o l_{g_{\lambda}}=d v o l_{g_{0}}$. Moreover, $g_{\lambda}$ is again $T$-invariant.

(iv) We say that a diffeomorphism $F: M \rightarrow M$ is T-preserving if conjugation by $F$ preserves $T \subset \operatorname{Diffeo}(\mathrm{M})$. In that case, we denote by $\Psi_{F}$ the automorphism of $\mathfrak{z}$ induced by conjugation by $F$. Note that if $F$ is $T$-equivariant then $\Psi_{F}=$ Id. Obviously, each $T$-preserving diffeomorphism $F$ of $M$ maps $T$-orbits to $T$-orbits and satisfies $F_{*}\left(Z^{*}\right)=\Psi_{F}(Z)^{*}$ for all $Z \in \mathfrak{z}$, where the vector fields $Z^{*}$ on $M$ are defined as in (i).

The following theorem gives a useful specialization of Theorem 1.3 and is the generalized analog of Theorem 1.6 from [Sch3], which corresponds to statement (i) below in the special case $\varphi=0$.

1.5 Theorem. In the context of Notation 1.4, let $\lambda, \lambda^{\prime}$ be two admissible $\mathfrak{z}$-valued 1-forms on $M$. Assume:

(*) For every $\mu \in \mathcal{L}^{*}$ there exists a T-equivariant $F_{\mu} \in \operatorname{Isom}\left(M, g_{0}\right)$ such that $\mu \circ \lambda=F_{\mu}^{*}\left(\mu \circ \lambda^{\prime}\right)$.

Let $\varphi \in C^{\infty}(M)$ be a potential function with the property that the isometries $F_{\mu}$ occurring in $(*)$ can be chosen such that $\varphi$ is invariant under each of them. Then we have:

(i) $\hbar^{2} \Delta_{g_{\lambda}}+\varphi$ and $\hbar^{2} \Delta_{g_{\lambda^{\prime}}}+\varphi$ are isospectral for each $\hbar \neq 0$; if $M$ has boundary then these operators are Dirichlet and Neumann isospectral.

(ii) If coincidentally there exists a T-preserving $\tau \in \operatorname{Isom}\left(M, g_{0}\right)$ such that, in the notation of $1.4(\mathrm{iv}), \Psi_{\tau} \circ \lambda=\tau^{*} \lambda^{\prime}$, but $\varphi \neq \tau^{*} \varphi$, then $\varphi$ and $\tau^{*} \varphi$ are 
isospectral potentials on $\left(M, g_{\lambda}\right)$; if $M$ has boundary then the two potentials are Dirichlet and Neumann isospectral.

Proof. (i) We claim that $\left(M, g_{\lambda}\right),\left(M, g_{\lambda^{\prime}}\right)$ and $\varphi, \psi:=\varphi$ satisfy the hypotheses of Theorem 1.3. Given a subtorus $W$ of codimension 1 in $T$ we choose $\mu \in \mathcal{L}^{*}$ such that $\operatorname{ker} \mu=T_{e} W$, and we choose a corresponding $F_{\mu}$ as in $(*)$. In the proof of Theorem 1.6 from [Sch3] (corresponding to the case $\varphi=0$ here) it was shown that $F_{W}:=F_{\mu}$ satisfies conditions (a) and (b) of Theorem 1.3; more precisely, (a) is immediate from $d v o l_{g_{\lambda}}=d v o l_{g_{0}}=d v o l_{g_{\lambda^{\prime}}}$, and (b) was deduced from condition $(*)$. It only remains to check that $F_{\mu}$ satisfies condition (c) of Theorem 1.3 (with $\psi=\varphi$ ); but this is already guaranteed by the assumptions on $\varphi$.

(ii) This follows immediately from (i) by noting that $\tau$ is an isometry from $\left(M, g_{\lambda}\right)$ to $\left(M, g_{\lambda^{\prime}}\right)$.

1.6 Corollary. Let $\lambda, \lambda^{\prime}$ be two admissible $\mathfrak{z}$-valued 1-forms on $M$ satisfying condition $(*)$ of Theorem 1.5. Moreover, let $\varphi \in C^{\infty}\left(M, \mathbb{R}_{+}\right)$be invariant under the action of $T$, and suppose that the isometries $F_{\mu}$ occurring in $(*)$ can be chosen such that $\varphi$ is invariant under each of them. Then we have:

(i) $\left(M, \varphi g_{\lambda}\right)$ and $\left(M, \varphi g_{\lambda^{\prime}}\right)$ are isospectral.

(ii) If coincidentally there exists a T-preserving $\tau \in \operatorname{Isom}\left(M, g_{0}\right)$ such that $\Psi_{\tau} \circ \lambda=\tau^{*} \lambda^{\prime}$, but $\tau^{*} \varphi \neq \varphi$, then the conformally equivalent metrics $\varphi g_{\lambda}$ and $\left(\tau^{*} \varphi\right) g_{\lambda}$ are isospectral.

Proof. (i) Note that by definition, $\varphi g_{\lambda}=(\varphi g)_{\lambda}$ (and similarly for $\lambda^{\prime}$ ). Thus the statement follows by applying Theorem 1.5(i) (with trivial potential) to $\lambda, \lambda^{\prime}$ and to $\varphi g_{0}$ instead of $g_{0}$.

(ii) This follows immediately from (i) by noting that $\tau$ is an isometry from $\left(M,\left(\tau^{*} \varphi\right) g_{\lambda}\right)$ to $\left(M, \varphi g_{\lambda^{\prime}}\right)$.

\section{$\S 2$ A NONISOMETRY PRINCIPLE}

In the following we let $\left(M, g_{0}\right), T, \mathfrak{z}$ be as in Notation 1.4 , and we consider two admissible $\mathfrak{z}$-valued 1 -forms $\lambda, \lambda^{\prime}$ on $M$ in the sense of Notation 1.4(ii). We define $\hat{M}$ as in Notation 1.2.

2.1 Notation and Remarks. ([Sch3])

(i) We denote by $\operatorname{Aut}_{g_{0}}^{T}(M)$ the group of all $T$-preserving diffeomorphisms $F$ of $M$ which, in addition, preserve the $g_{0}$-norm of vectors tangent to the $T$-orbits and induce an isometry, denoted $\bar{F}$, of $\left(\hat{M} / T, g_{0}^{T}\right)$. We denote the corresponding group of induced isometries by $\overline{\operatorname{Aut}}_{g_{0}}^{T}(M) \subset \operatorname{Isom}\left(\hat{M} / T, g_{0}^{T}\right)$.

(ii) We define $\mathcal{D}:=\left\{\Psi_{F} \mid F \in \operatorname{Aut}_{g_{0}}^{T}(M)\right\} \subset \operatorname{Aut}(\mathfrak{z})$ (see Notation 1.4(iv)). Note that $\mathcal{D}$ is discrete because it is a subgroup of the discrete group $\{\Psi \in$ $\operatorname{Aut}(\mathfrak{z}) \mid \Psi(\mathcal{L})=\mathcal{L}\}$, where $\mathcal{L}$ is the lattice $\operatorname{ker}(\exp : \mathfrak{z} \rightarrow T)$. 
(iii) Let $\omega_{0}$ denote the connection form on $\hat{M}$ associated with $g_{0}$; i.e., for each $x \in \hat{M}$ the horizontal space $\operatorname{ker}\left(\left.\omega_{0}\right|_{T_{x}} \hat{M}\right)$ is the $g_{0}$-orthogonal complement of $\mathfrak{z} x$ in $T_{x} \hat{M}$ and $\omega_{0}\left(Z_{x}^{*}\right)=Z$ for $Z \in \mathfrak{z}$. Then the connection form on $\hat{M}$ associated with $g_{\lambda}$ is given by $\omega_{\lambda}:=\omega_{0}+\lambda$. Let $\Omega_{\lambda}$ denote the curvature form on $\hat{M} / T$ associated with the connection form $\omega_{\lambda}$ on $\hat{M}$. We have $\pi^{*} \Omega_{\lambda}=d \omega_{\lambda}$ because $T$ is abelian.

(iv) Since $\lambda$ is $T$-invariant and horizontal it induces a $\mathfrak{z}$-valued 1-form $\bar{\lambda}$ on $\hat{M} / T$. We conclude from $\pi^{*} \Omega_{\lambda}=d \omega_{\lambda}=d \omega_{0}+d \lambda$ that $\Omega_{\lambda}=\Omega_{0}+d \bar{\lambda}$. In particular, $\Omega_{\lambda}$ and $\Omega_{0}$ differ by an exact $\mathfrak{z}$-valued 2 -form.

2.2 Proposition ([Sch3]). Let $\lambda, \lambda^{\prime}$ be admissible $\mathfrak{z}$-valued 1 -forms on $M$ satisfying the following two conditions:

$$
\Omega_{\lambda} \notin \mathcal{D} \circ \overline{\operatorname{Aut}}_{g_{0}}^{T}(M)^{*} \Omega_{\lambda^{\prime}}
$$

No nontrivial 1-parameter group in $\overline{\operatorname{Aut}}_{g_{0}}^{T}(M)$ preserves $\Omega_{\lambda^{\prime}}$.

Then $\left(M, g_{\lambda}\right)$ and $\left(M, g_{\lambda^{\prime}}\right)$ are not isometric.

Remark 2.3. (i) For our examples of conformally equivalent isospectral metrics on balls and spheres that we will construct in Sections 4 by applying Proposition 1.6, we will use the nonisometry result above with $\varphi g_{0}$ playing the role of $g_{0}$. We will not be able to apply this result to the Lie group examples in Section 3, however, and thus will prove the non-isometry of those examples by a different technique.

(ii) To construct isospectral potentials via Theorem 1.5, we may choose to use the same functions $\varphi$ as for the examples of conformally equivalent isospectral metrics arising from Corollary 1.6 (although Theorem 1.5 permits a wider choice of potentials). Once we show that a pair of conformally equivalent isospectral metrics $\varphi g_{\lambda}$ and $\left(\tau^{*} \varphi\right) g_{\lambda}$ constructed as in Corollary 1.6(ii) is nonisometric, it will follow immediately that the isospectral (by Theorem 1.5) potentials $\varphi$ and $\tau^{*} \varphi$ for the Schrödinger operator on $\left(M, g_{\lambda}\right)$ are not congruent under any isometry of $\left(M, g_{\lambda}\right)$. Indeed, if the potentials were congruent, then the metrics $\varphi g_{\lambda}$ and $\left(\tau^{*} \varphi\right) g_{\lambda}$ would be isometric.

\section{$\S 3$ EXAmples I: LiE GROUPS}

The examples in this section are modelled after the construction of isospectral left invariant Riemannian metrics on compact Lie groups given in [Sch2].

Let $\mathfrak{g}$ be a Lie algebra. Recall that $\operatorname{ad}_{\mathfrak{g}}$ is a subalgebra of $\operatorname{Der}(\mathfrak{g})$, the algebra of derivations of $\mathfrak{g}$. The connected subgroup of $\operatorname{Aut}(\mathfrak{g})$ with Lie algebra $\operatorname{ad}(\mathfrak{g})$ is denoted $\operatorname{Inn}(\mathfrak{g})$ and is called the group of inner automorphisms of $\mathfrak{g}$. If $G$ is any connected Lie group with Lie algebra $\mathfrak{g}$, then $\operatorname{Inn}(\mathfrak{g})=\operatorname{Ad}_{G}$. In particular, if $G$ is a matrix group and thus $\mathfrak{g}$ is a matrix algebra, then $\operatorname{Inn}(\mathfrak{g})$ consists of all maps of $\mathfrak{g}$ given by conjugation by matrices in $G$. 
Recall also that a Lie algebra is said to be compact if it is the Lie algebra of a compact Lie group.

3.1 Definition. Let $\mathfrak{g}$ be a compact Lie algebra, let $\mathfrak{z}$ be a real vector space with an inner product, and let $j, j^{\prime}: \mathfrak{z} \rightarrow \mathfrak{g}$ be linear maps.

(i) $j$ and $j^{\prime}$ will be said to be isospectral if for each $Z \in \mathfrak{z}$, there exists $\alpha_{Z} \in \operatorname{Inn}(\mathfrak{g})$ such that $j^{\prime}(Z)=\alpha_{Z}(j(Z)$ ). (We will focus on matrix algebras, in which case this condition may be rewritten as $j^{\prime}(Z)=\operatorname{Ad}\left(A_{Z}\right)(j(Z))=A_{Z} j(Z) A_{Z}^{-1}$ for some $A_{Z}$ in the associated connected matrix group.)

(ii) $j$ and $j^{\prime}$ will be said to be equivalent if there exists an automorphism $\Phi$ of $\mathfrak{g}$ (not necessarily inner) and an orthogonal linear map $C$ of $\mathfrak{z}$ such that $j^{\prime}(Z)=$ $\Phi(j(C(Z)))$ for all $Z \in \mathfrak{z}$. In this case we will say that the pair $(\Phi, C)$ is an equivalence between $j$ and $j^{\prime}$.

Remarks. The notion of isospectrality and of equivalence of maps $j, j^{\prime}: \mathfrak{z} \rightarrow \mathfrak{g}$ was first introduced in [GW2] in case $\mathfrak{g}=\mathfrak{s o}(m)$ and in $[\mathrm{Sch} 2,3]$ in case $\mathfrak{g}=\mathfrak{s u}(m)$. Our notions of isospectrality and equivalence in case $\mathfrak{g}=\mathfrak{s o}(\mathrm{m})$ differ slightly from those in [GW2]: the conjugating elements $A_{Z} \in \mathrm{SO}(m)$ in Definition 3.1(i) were allowed to lie instead in $\mathrm{O}(m)$ in [GW2]. In the notion of equivalence used in [GW2], the automorphism $\Phi$ was required to lie in $\operatorname{Ad}_{\mathrm{O}(m)}$. Since $\operatorname{Aut}(\mathfrak{s o}(m))=\operatorname{Ad}_{\mathrm{O}(m)}$ except when $m=4$ or $m=8$, the two notions of equivalence actually agree except in these special dimensions.

Isospectral maps $j$ and $j^{\prime}$ will be used in various constructions of isospectral Riemannian manifolds. In general, equivalence of the maps $j$ and $j^{\prime}$ will imply isometry of the resulting manifolds; the converse will hold under generic conditions.

3.2 Proposition. (i) [GW2] Let $m$ be any positive integer other than 1,2,3,4, or 6 . Let $\mathcal{J}$ be the real vector space consisting of all linear maps $j: \mathbb{R}^{2} \rightarrow \mathfrak{s o}(m)$. Then there is a Zariski open subset $\mathcal{O}$ of $\mathcal{J}$ (i.e., $\mathcal{O}$ is the complement of the zero locus of some nonzero polynomial function on $\mathcal{J}$ ) such that each $j \in \mathcal{O}$ belongs to a d-parameter family of isospectral, inequivalent elements of $\mathcal{J}$. Here $d \geq m(m-1) / 2-[m / 2]([m / 2]+2)>1$. In particular, $d$ is of order at least $O\left(m^{2}\right)$.

(ii) [Sch2] Let $m \geq 3$. Let $\mathcal{J}$ be the real vector space consisting of all linear maps $j: \mathbb{R}^{2} \rightarrow \mathfrak{s u}(m)$. Then there is a Zariski open subset $\mathcal{O}$ of $\mathcal{J}$ such that each $j \in \mathcal{O}$ belongs to a continuous family of isospectral, inequivalent elements of $\mathcal{J}$.

Remarks. (i) The article [GW2] also gives an example of a continuous family of isospectral, inequivalent maps from $\mathbb{R}^{2}$ to $\mathfrak{s o}(6)$.

(ii) The two parts of the proposition were proven in [GW2] and [Sch2,3]. In the case of $\mathfrak{s o}(\mathrm{m})$ in [GW2], the notions of isospectrality and equivalence defined in the remark following Definition 3.1 were used. However, the proposition remains true if we instead define isospectrality as in Definition 3.1. Indeed, as pointed 
out in [Sch2], isospectrality (in the sense of [GW2]) of a continuous family $j_{t}$ as in Proposition 3.2(i) means that on each $Z \in \mathbb{R}^{2}$, the path $t \rightarrow j_{t}(Z)$ lies in a single $\operatorname{Ad}_{\mathrm{O}(m)}$-orbit of $j_{0}(Z)$ and thus, by continuity, the path in fact lives in a

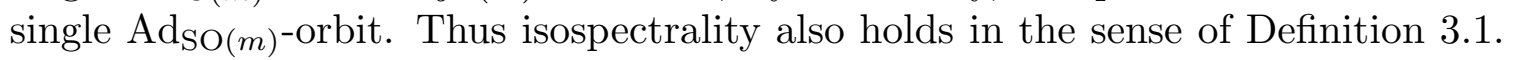
A similar argument may be used to show that inequivalence in the sense of [GW2] of the continuous family $\left\{j_{t}\right\}$ implies inequivalence in the sense of Definition 3.1.

(iii) In Section 5, we will place various genericity conditions on the maps $j$. The assertions of Proposition 3.2 remain true with these genericity conditions, as one can see from the original proofs.

The following lemma is immediate.

3.3 Lemma. Let $\mathfrak{k}$ be a compact Lie algebra, and let $j_{1}, j_{2}: \mathfrak{z} \rightarrow \mathfrak{k}$ be isospectral linear maps as in Definition 3.1. Let $\mathfrak{g}$ be a compact Lie algebra containing $\mathfrak{k} \oplus \mathfrak{k}$ as a subalgebra. Define $j, j^{\prime}: \mathfrak{z} \rightarrow \mathfrak{k} \oplus \mathfrak{k} \subset \mathfrak{g}$ by $j(Z)=\left(j_{1}(Z), j_{2}(Z)\right)$ and $j^{\prime}(Z)=$ $\left(j_{2}(Z), j_{1}(Z)\right)$. Then

$$
j^{\prime}(Z)=\left(\alpha_{Z}, \alpha_{Z}^{-1}\right)(j(Z))
$$

for each $Z \in \mathfrak{z}$, where $\alpha_{Z}$ is given as in Definition 3.1 with respect to the pair of isospectral maps $j_{1}, j_{2}$. Thus $j$ and $j^{\prime}$ are isospectral.

3.4 Notation and Remarks. Let $G$ be a compact Lie group with Lie algebra $\mathfrak{g}$, and let $g_{0}$ be a bi-invariant Riemannian metric on $G$. Let $H<G$ be a closed connected subgroup of $G$ with Lie algebra $\mathfrak{h}$, and let $T<G$ be a torus with Lie algebra $\mathfrak{z}$. Suppose that $[\mathfrak{z}, \mathfrak{h}]=0$, and that $\mathfrak{z}$ is $g_{0}$-orthogonal to $\mathfrak{h}$ in $\mathfrak{z}$.

(i) For any linear map $j: \mathfrak{z} \rightarrow \mathfrak{h}$ we define an associated left invariant $\mathfrak{z}$-valued 1-form $\lambda^{j}$ on $G$ by letting $g_{0}\left(\lambda^{j}(X), Z\right)=g_{0}(X, j(Z))$ for all $Z \in \mathfrak{z}$ and $X \in \mathfrak{g}$ (in other words, $\lambda^{j}: \mathfrak{g} \rightarrow \mathfrak{z}$ is the $g_{0}$-transpose of $j: \mathfrak{z} \rightarrow \mathfrak{h} \subset \mathfrak{g}$ ).

(ii) We note that $\lambda^{j}$ is admissible in the sense of 1.4 with respect to the action of $T$ on $G$ from the right. In fact, $\lambda^{j}$ vanishes on the $g_{0}$-orthogonal complement of $\mathfrak{h}$ and thus, in particular, on $\mathfrak{z}$. The left invariant vector fields $Z$ with $Z \in \mathfrak{z}$ are just the vector fields $Z^{*}$ (as in 1.4) induced by the right action of $T$. Moreover, $\lambda^{j}$ is invariant under this action: Since $[\mathfrak{z}, \mathfrak{h}]=0$, the Lie algebra $\mathfrak{h}$ is $g_{0}$-orthogonal to $[\mathfrak{z}, \mathfrak{g}]$; therefore, $\lambda^{j}$ vanishes on $[\mathfrak{z}, \mathfrak{g}]$ and is hence invariant under $\operatorname{Ad}_{T}$. Thus $\lambda^{j}$ is not only left invariant but also right invariant under $T$.

(iii) Since $\lambda^{j}$ is admissible with respect to the right action of $T$, we can define the associated metric $g_{\lambda^{j}}$ on $G$ as in Notation 1.4. Note that $g_{\lambda^{j}}$ is left invariant (and right invariant under $T$ ).

3.5 Lemma [Sch2]. In the notation of 3.1 and 3.4 , suppose that $j, j^{\prime}: \mathfrak{z} \rightarrow \mathfrak{h}$ are isospectral. Let $\lambda, \lambda^{\prime}: \mathfrak{g} \rightarrow \mathfrak{z}$ be the associated left-invariant 1-forms. Given a linear functional $\mu$ on $\mathfrak{z}$, let $Z \in \mathfrak{z}$ satisfy $\mu(W)=g_{0}(W, Z)$ for all $W \in \mathfrak{z}$. Define 
$F_{\mu}:=L_{A_{Z}} \circ R_{A_{Z}}^{-1} \in \operatorname{Aut}(G)$, where $A_{Z} \in H$ is such that $j^{\prime}(Z)=\operatorname{Ad}\left(A_{Z}\right)(j(Z))$ (the existence of $A_{Z}$ follows from Definition 3.1). Then

$$
\mu \circ \lambda=F_{\mu}^{*}\left(\mu \circ \lambda^{\prime}\right)
$$

moreover, $F_{\mu}$ is T-equivariant because $H$ and $T$ commute, and is a $g_{0}$-isometry because $g_{0}$ is bi-invariant. In particular, the associated Riemannian metrics $g_{\lambda}$ and $g_{\lambda^{\prime}}$ on $G$ are isospectral by Theorem 1.5(i) (with $\varphi=0$ ).

3.6 Theorem. Let $G$ be a compact Lie group with Lie algebra $\mathfrak{g}$, let $H$ be a compact Lie subgroup with Lie algebra of the form $\mathfrak{h}=\mathfrak{k} \oplus \mathfrak{k}$ for some Lie algebra $\mathfrak{k}$, and let $T<G$ be a torus with Lie algebra $\mathfrak{z}$. Suppose that $[\mathfrak{z}, \mathfrak{h}]=0$, and that $\mathfrak{z}$ is orthogonal to $\mathfrak{h}$ with respect to a bi-invariant Riemannian metric $g_{0}$ on $G$. Let $j_{1}, j_{2}: \mathfrak{z} \rightarrow \mathfrak{k}$ be isospectral linear maps as in 3.1. Define $j: \mathfrak{z} \rightarrow \mathfrak{k} \oplus \mathfrak{k}=\mathfrak{h}$ by $j(Z)=\left(j_{1}(Z), j_{2}(Z)\right)$. Denote by $g_{\lambda}$ the associated left invariant metric on $G$ defined as in 3.4, where $\lambda:=\lambda^{j}$. Let $\varphi$ be a smooth function on $G$ which is invariant under conjugation by elements of $H$. Suppose that there exists an isometric automorphism $\tau$ of $\left(G, g_{0}\right)$ such that $\left.\tau\right|_{T}=I d$ and such that $\tau_{*}$ restricts to the map $(X, Y) \mapsto(Y, X)$ on $\mathfrak{k} \oplus \mathfrak{k}=\mathfrak{h} \subset \mathfrak{g}$. Then:

(i) $\varphi$ and $\tau^{*} \varphi$ are isospectral potentials on $\left(G, g_{\lambda}\right)$.

(ii) If, in addition, $\varphi$ is positive and right invariant under $T$, then $\varphi g_{\lambda}$ and $\left(\tau^{*} \varphi\right) g_{\lambda}$ are conformally equivalent isospectral metrics on $G$.

Proof. Define $j^{\prime}: \mathfrak{z} \rightarrow \mathfrak{k} \oplus \mathfrak{k}$ by $j^{\prime}(Z):=\left(j_{2}(Z), j_{1}(Z)\right)$. By Lemma $3.3, j$ and $j^{\prime}$ are isospectral. Let $\lambda^{\prime}:=\lambda^{j^{\prime}}$. By Lemma 3.5, $\lambda$ and $\lambda^{\prime}$ satisfy condition $(*)$ of Theorem 1.5 with respect to the right action of $T$ on $G$, with each $F_{\mu}$ given as conjugation by an element of $H$. Since $\tau$ is a $T$-equivariant $g_{0}$-isometry and obviously satisfies $\tau_{*} j(Z)=j^{\prime}(Z)$ for all $Z \in \mathfrak{z}$, if follows immediately from the definition of $\lambda$ and $\lambda^{\prime}$ that $\tau^{*} \lambda^{\prime}=\lambda$. Thus statements (i) and (ii) follow from Theorem 1.5(ii) and Corollary 1.6, respectively.

3.7 Examples. (i) Given a vector space $\mathfrak{z}$, say of dimension $r$, we may realize $\mathfrak{z}$ as the Lie algebra of a maximal torus $T$ in $\mathrm{SO}(2 r)$. Given isospectral maps $j_{1}, j_{2}: \mathfrak{z} \rightarrow$ $\mathfrak{s o}(m)=: \mathfrak{k}$, let $G=\mathrm{SO}(2 m+2 r)$ with a bi-invariant Riemannian metric $g_{0}$. Then $G$ contains $\mathrm{SO}(m) \times \mathrm{SO}(m) \times \mathrm{SO}(2 r)$ as the subgroup formed by three diagonal blocks, which in turn contains $\mathrm{SO}(m) \times \mathrm{SO}(m) \times T$. Set $H=\mathrm{SO}(m) \times \mathrm{SO}(m)$. The map $(p, q) \mapsto(q, p)$ of $H$ extends to an inner automorphism - hence an isometry - $\tau$ of $G$ (namely, conjugation by the orthogonal map of $\mathbb{R}^{2 m+2 r}$ interchanging the first $m$ coordinates with the next $m$ coordinates). Thus Theorem 3.6 yields an associated left invariant metric $g_{\lambda}$ on $G$ and, for any smooth function $\varphi$ on $G$ which is invariant under conjugation by elements of $H$, a pair of isospectral potentials for the Schrödinger operator on $\left(G, g_{\lambda}\right)$. Choosing $\varphi$ also to be positive and right invariant under $T$, we further obtain a pair of isospectral metrics conformally equivalent to $g_{\lambda}$. In particular, Proposition 3.2 and the subsequent remark allow us to 
construct isospectral potentials and conformally equivalent isospectral metrics on $\mathrm{SO}(2 m+4)$ for all $m \geq 5$. (Although Proposition 3.2 yields continuous families of isospectral $j$-maps, the construction in Theorem 3.6 requires us to work with pairs of isospectral maps. Thus we do not obtain continuous isospectral deformations of potentials or of conformally equivalent metrics by this construction.)

(ii) Consider the two-fold covering $\tilde{G}=\operatorname{Spin}(2 m+2 r)$ of $\mathrm{SO}(2 m+2 r)$. Letting $\tilde{H}$ be the connected subgroup of $\tilde{G}$ with Lie algebra $\mathfrak{h}=\mathfrak{s o}(m) \oplus \mathfrak{s o}(m)$ and $\tilde{T}$ the toral subgroup with Lie algebra $\mathfrak{z} \subset \mathfrak{s o}(2 r)$, and letting $j_{1}, j_{2}: \mathfrak{z} \rightarrow \mathfrak{k}=\mathfrak{s o}(m)$ be isospectral linear maps, we then obtain, exactly as in Example (i), an associated metric $g_{\lambda}$ on $\tilde{G}$ and, for any function $\varphi$ on $\tilde{G}$ which is invariant under conjugation by elements of $\tilde{H}$, a pair of isospectral potentials for the Schrödinger operator on $\left(\tilde{G}, g_{\lambda}\right)$. Note for that purpose that the automorphism $\tau$ of $S O(2 m+2 r)$ lifts to an automorphism $\tilde{\tau}$ of $\tilde{G}$. If $\varphi$ is also positive and right invariant under $\tilde{T}$ then we further obtain a pair of isospectral metrics conformally equivalent to $g_{\lambda}$. Thus we get pairs of isospectral potentials and conformally equivalent isospectral metrics on $\operatorname{Spin}(2 m+4)$ for all $m \geq 5$.

(iii) In the notation of Example (i), we may also realize $\mathfrak{z}$ as a maximal torus in $\mathrm{SU}(r+1)$. The analogous construction with $\mathfrak{k}=\mathfrak{s u}(m)$ then yields, for each pair of isospectral maps $j_{1}, j_{2}: \mathfrak{z} \rightarrow \mathfrak{s u}(m)=: \mathfrak{k}$, an associated left invariant Riemannian metric $g_{\lambda}$ on $G:=\mathrm{SU}(2 m+r+1)$ and, for appropriately chosen $\varphi$, isospectral potentials on $\left(G, g_{\lambda}\right)$ and isospectral metrics on $G$ conformally equivalent to $g_{\lambda}$. In particular, applying Proposition 3.2(ii), we obtain such isospectral potentials and conformally equivalent metrics on $\mathrm{SU}(2 m+3)$ for all $m \geq 3$.

3.8 Remark. In 5.1 we will prove nontriviality of the above examples from 3.7 for a particular choice of $\varphi$ under the hypothesis that $j_{1}$ is not equivalent to $j_{2}$ and under certain genericity conditions on $j$.

\section{$\S 4$ Examples II: Spheres AND Balls}

Let $r \geq 2$, and let $T$ be the $r$-dimensional torus $\mathbb{R}^{r} / \mathcal{L}$ with $\mathcal{L}=(2 \pi \mathbb{Z})^{r}$. Let $\left\{Z_{1}, \ldots, Z_{r}\right\}$ be the standard basis of its Lie algebra $\mathfrak{z}=\mathbb{R}^{r}$.

\subsection{Notation.}

(i) For any given $n$, we consider the action of $T=\left(S^{1}\right)^{r}$ on $\mathbb{R}^{n} \oplus \mathbb{C}^{r}$ which is induced by the canonical action of $S^{1} \times \ldots \times S^{1}$ on $\mathbb{C} \times \ldots \times \mathbb{C}$ given by multiplication in each factor.

(ii) Given any $\mathfrak{z}$-valued 1 -form $\lambda$ on $\mathbb{R}^{n}$, its trivial extension to $\mathbb{R}^{n} \oplus \mathbb{C}^{r}$ (again denoted $\lambda$ ) is admissible in the sense of Notation 1.4 with respect to the action of $T$ defined above. Let $\left(B, g_{0}\right)$, respectively $\left(S, g_{0}\right)$, be the standard unit ball, respectively sphere, in $\mathbb{R}^{n} \oplus \mathbb{C}^{r} \cong \mathbb{R}^{n+2 r}$. We continue to denote by $\lambda$ the restriction, respectively pullback, of $\lambda$ to $B$, respectively $S$. Then $\lambda$ is admissible with respect to the induced action of $T$ and thus defines a metric $g_{\lambda}$ on $B$ and on $S$. 
4.2 Definition. Two $\mathfrak{z}$-valued 1-forms $\nu, \nu^{\prime}$ on $\mathbb{R}^{m}$ are called isospectral if for each $\mu \in \mathcal{L}^{*}$ there exists $A_{\mu} \in \mathrm{O}(m)$ such that $\mu \circ \nu=A_{\mu}^{*}(\mu \circ \nu)$.

4.3 Theorem. Let $n=2 m$, and let $M$ denote either the ball $B$ or the sphere $S$ in $\mathbb{R}^{n+2 r} \cong \mathbb{R}^{n} \oplus \mathbb{C}^{r}$. Let $\nu, \nu^{\prime}$ be isospectral $\mathfrak{z}$-valued 1 -forms on $\mathbb{R}^{m}$. Define a $\mathfrak{z}$-valued 1 -form $\lambda$ on $\mathbb{R}^{n}=\mathbb{R}^{m} \oplus \mathbb{R}^{m}$ by

$$
\lambda_{(p, q)}(X, Y):=\nu_{p}(X)+\nu_{q}^{\prime}(Y) .
$$

Denote by $g_{\lambda}$ the associated metric on $M$ as in Notation 4.1 (ii). Let $\psi: \mathbb{R}^{m} \rightarrow \mathbb{R}$ be a smooth radial function and define $\varphi_{1}, \varphi_{2}: \mathbb{R}^{m} \oplus \mathbb{R}^{m} \oplus \mathbb{C}^{r} \rightarrow \mathbb{R}$ by $\varphi_{i}\left(p_{1}, p_{2}, u\right):=$ $\psi\left(p_{i}\right)$ for $i=1,2$. Then

(i) $\varphi_{1}$ and $\varphi_{2}$ are isospectral potentials on $\left(M, g_{\lambda}\right)$.

(ii) If $\psi$ is strictly positive, then $\varphi_{1} g_{\lambda}$ and $\varphi_{2} g_{\lambda}$ are isospectral conformally equivalent Riemannian metrics on $M$.

Proof. We apply the criteria of Theorem 1.5 and Corollary 1.6. Define a second $\mathfrak{z}$-valued 1-form $\lambda^{\prime}$ on $\mathbb{R}^{n}=\mathbb{R}^{m} \oplus \mathbb{R}^{m}$ by

$$
\lambda_{(p, q)}^{\prime}(X, Y):=\nu_{p}^{\prime}(X)+\nu_{q}(Y) .
$$

Let $g_{\lambda^{\prime}}$ be the associated Riemannian metric on $M$. Given any $\mu \in \mathcal{L}^{*}$, choose $A_{\mu} \in \mathrm{O}(m)$ as in Definition 4.2. Then the map

$$
F_{\mu}: \mathbb{R}^{m} \oplus \mathbb{R}^{m} \oplus \mathbb{C}^{r} \ni(p, q, u) \mapsto\left(A_{\mu} p, A_{\mu}^{-1} q, u\right) \in \mathbb{R}^{m} \oplus \mathbb{R}^{m} \oplus \mathbb{C}^{r}
$$

restricts to a $T$-equivariant isometry of $\left(M, g_{0}\right)$ satisfying $\mu \circ \lambda=F_{\mu}^{*}\left(\mu \circ \lambda^{\prime}\right)$. Moreover, the map

$$
\tau: \mathbb{R}^{m} \oplus \mathbb{R}^{m} \oplus \mathbb{C}^{r} \ni(p, q, u) \mapsto(q, p, u) \in \mathbb{R}^{m} \oplus \mathbb{R}^{m} \oplus \mathbb{C}^{r}
$$

also restricts to a $T$-equivariant isometry of $\left(M, g_{0}\right)$ and satisfies $\lambda=\tau^{*} \lambda^{\prime}$.

We let $\varphi_{1}$ play the role of $\varphi$ in the notation of 1.5 and 1.6, and observe that $\varphi_{2}=\tau^{*} \varphi_{1}$. Since $\psi$ is radial, $\varphi_{1}$ is invariant under each $F_{\mu}$. Thus Theorem 3.8 follows from Theorem 1.5 and Corollary 1.6.

4.4 Example. Our first family of examples is modelled after the construction of isospectral balls and spheres in [Go3] (see Remark 4.5 below).

Let $j, j^{\prime}: \mathfrak{z} \rightarrow \mathfrak{s o}(m)$ be isospectral linear maps, where we allow the weaker notion of isospectrality given in the remark following Definition 3.1. Define two $\mathfrak{z}$-valued 1 -forms $\nu, \nu^{\prime}$ on $\mathbb{R}^{m}$ by

$$
\left\langle\nu^{(\prime)}\left(X_{p}\right), Z\right\rangle=\left\langle j^{(\prime)}(Z) p, X\right\rangle
$$


for $X_{p} \in T_{p} \mathbb{R}^{m}$ and $Z \in \mathfrak{z}$, where $\langle$,$\rangle denotes the standard inner products both$ on $\mathbb{R}^{m}$ and on $\mathfrak{z}=\mathbb{R}^{k}$. Then $\nu$ and $\nu^{\prime}$ are isospectral in the sense of Definition 4.2. In fact, let $Z \in \mathfrak{z}$ be the vector dual to $\mu$ with respect to the inner product $\langle$,$\rangle on$ z. Choose $A_{Z} \in \mathrm{O}(m)$ as in the remark following Definition 3.1; then $A_{\mu}:=A_{Z}$ will satisfy $\mu \circ \nu=A_{\mu}^{*}\left(\mu \circ \nu^{\prime}\right)$.

Recall from Proposition 3.2 that for $r=2$, i.e. $\mathfrak{z}=\mathbb{R}^{2}$, and each $m \geq 5$ there do exist pairs $j, j^{\prime}: \mathfrak{z} \rightarrow \mathfrak{s o}(m)$ as above. Using the corresponding forms $\nu, \nu^{\prime}$ for constructing $\lambda$ in Theorem 4.3, we obtain a metric $g_{\lambda}$, pairs of isospectral metrics conformally equivalent to $g_{\lambda}$, and pairs of potentials which are isospectral with respect to $g_{\lambda}$ on each of the spheres $S^{2 m+3 \geq 13}$ and the balls $B^{2 m+4 \geq 14}$.

4.5 Remark. In 5.2 we will show that the examples from 4.4 with $\mathfrak{z}=\mathbb{R}^{2}$ are nontrivial provided that

(i) the maps $j, j^{\prime}$ are not equivalent in the sense of Definition 3.1,

(ii) $j$ and $j^{\prime}$ are generic in the sense that no nonzero element of $\mathfrak{s o}(m)$ commutes with both $j\left(Z_{1}\right), j\left(Z_{2}\right)$, or with both $j^{\prime}\left(Z_{1}\right), j^{\prime}\left(Z_{2}\right)$, and

(iii) the restriction to $\overline{B_{1}(0)} \subset \mathbb{R}^{m}$ of the smooth radial function $\psi: \mathbb{R}^{m} \rightarrow \mathbb{R}_{+}$ attains its maximum precisely in $p=0$.

Remark. In the notation of Theorem 4.3 and Example 4.4 above, the isospectral spheres and balls from [Go3] were just the unit spheres, respectively balls, in $\mathbb{R}^{m} \oplus$ $\mathbb{C}^{r}$, endowed with the associated isospectral metrics $g_{\nu}$ and $g_{\nu^{\prime}}$.

Note that in Example 4.4, we had $r \geq 2$ and $m \geq 5$, where $r$ and $m$ are the dimensions used in Theorem 4.3. We now give an example with $r=2$ and $m=3$, yielding conformally equivalent isospectral metrics and isospectral potentials on $S^{9} \subset \mathbb{R}^{3} \oplus \mathbb{R}^{3} \oplus \mathbb{C}^{2}$ and on $B^{10}$. The approach is related to certain constructions from $[$ Sch2,3].

\subsection{Example.}

Let $r=2$, thus $\mathfrak{z}=\mathbb{R}^{2}$. Let $\operatorname{Sym}_{0}\left(\mathbb{R}^{3}\right)$ denote the space of symmetric traceless real $3 \times 3$-matrices. For each linear map $c: \mathfrak{z} \cong \mathbb{R}^{2} \rightarrow \operatorname{Sym}_{0}\left(\mathbb{R}^{3}\right)$ we define a $\mathfrak{z}$-valued 1 -form $\nu$ on $\mathbb{R}^{3}$ by letting

$$
\left\langle\nu\left(X_{p}\right), Z\right\rangle=\langle c(Z) p \times p, X\rangle
$$

for all $X \in T_{p} \mathbb{R}^{3}$ and $Z \in \mathfrak{z}$, where $\langle$,$\rangle denotes the standard euclidean inner$ products on both $\mathbb{R}^{3}$ and on $\mathfrak{z}$, and $\times$ denotes the vector product in $\mathbb{R}^{3}$.

The key observation is that there exist pairs of linear maps $c, c^{\prime}: \mathfrak{z} \rightarrow \operatorname{Sym}_{0}\left(\mathbb{R}^{3}\right)$ such that

1.) $c$ and $c^{\prime}$ are isospectral, that is, for each $Z \in \mathfrak{z}$ there exists $E_{Z} \in \mathrm{SO}(3)$ such that $c^{\prime}(Z)=E_{Z} c(Z) E_{Z}^{-1}$

2.) $c$ and $c^{\prime}$ are not equivalent, where being equivalent means: There exists $E \in \mathrm{O}(3)$ and $C \in \mathrm{O}(2)$ such that $c^{\prime}(Z)=E c(C(Z)) E^{-1}$ for all $Z \in \mathfrak{z}$; 
3.) $c$ and $c^{\prime}$ are generic, that is, no nonzero element of $\mathfrak{s o}(3)$ commutes with both $c\left(Z_{1}\right)$ and $c\left(Z_{2}\right)$, and the analogous statement holds for $c^{\prime}$.

An explicit example of a pair $c, c^{\prime}$ satisfying the conditions above is given by

$$
c\left(Z_{1}\right)=c^{\prime}\left(Z_{1}\right)=\left(\begin{array}{ccc}
-1 & 0 & 0 \\
0 & 0 & 0 \\
0 & 0 & 1
\end{array}\right), \quad c\left(Z_{2}\right)=\left(\begin{array}{ccc}
0 & 1 / \sqrt{2} & 0 \\
1 / \sqrt{2} & 0 & 1 / \sqrt{2} \\
0 & 1 / \sqrt{2} & 0
\end{array}\right), \quad c^{\prime}\left(Z_{2}\right)=\left(\begin{array}{lll}
0 & 0 & 1 \\
0 & 0 & 0 \\
1 & 0 & 0
\end{array}\right)
$$

(compare [Sch3], Proposition 3.3.6; actually $c\left(Z_{2}\right)$ and $c^{\prime}\left(Z_{2}\right)$ were rescaled by $\sqrt{2}$ there, but this does not affect any of the three properties above).

It is easy to check that condition 1.) implies isospectrality (in the sense of 4.2) of

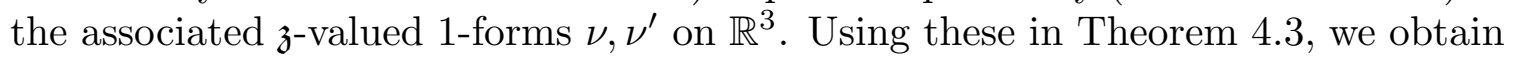
pairs of isospectral potentials and pairs of conformally equivalent isospectral metrics on $S=S^{9} \subset \mathbb{R}^{3} \oplus \mathbb{R}^{3} \oplus \mathbb{C}^{2}$, resp. on $B=B^{10}$.

In 5.2 we will prove the corresponding nontriviality statements, provided that the restriction to $\overline{B_{1}(0)} \subset \mathbb{R}^{3}$ of the smooth radial function $\psi: \mathbb{R}^{3} \rightarrow \mathbb{R}_{+}$attains its maximum precisely in $p=0$.

Having thus obtained examples of conformally equivalent isospectral metrics and isospectral potentials on $S^{9}$ and $B^{10}$, we do a final twist in order to obtain such examples even on $S^{7}$ and $B^{8}$. The idea is to lift the forms $\nu, \nu^{\prime}$ used in Example 4.6 from $\mathbb{R}^{3}$ to $\mathbb{C}^{2}$ via the Hopf projection. The new $\lambda=\nu \oplus \nu^{\prime}$ will live on $\mathbb{C}^{2} \oplus \mathbb{C}^{2} \cong \mathbb{R}^{8}$ instead of $\mathbb{R}^{3} \oplus \mathbb{R}^{3}$. In return, the third component of our previous ambient space $\mathbb{R}^{3} \oplus \mathbb{R}^{3} \oplus \mathbb{C}^{2}$ will become unnecessary because we will use a different torus action, living on $\mathbb{C}^{2} \oplus \mathbb{C}^{2}$ itself. This construction is related to a construction from [Sch3] of isospectral metrics on $S^{5} \subset \mathbb{C}^{2} \oplus \mathbb{C}$ and on $B^{6}$.

First we need some preparations.

4.7 Notation. (i) Let $r=2$, thus $\mathfrak{z}=\mathbb{R}^{2}$ and $T=\mathbb{R}^{2} /(2 \pi \mathbb{Z})^{2}$. For any $m \in \mathbb{N}$ we identify the real vector spaces $\mathbb{C}^{m}$ and $\mathbb{R}^{2 m}$ via the linear isomorphism which sends $\left\{e_{1}, i e_{1}, \ldots, e_{m}, i e_{m}\right\}$ (in this order) to the standard basis of $\mathbb{R}^{2 m}$, where $\left\{e_{1}, \ldots, e_{m}\right\}$ denotes the standard basis of $\mathbb{C}^{m}$. We let the torus $T$ act on $\mathbb{C}^{m} \oplus \mathbb{C}^{m}$ by

$$
\exp \left(a Z_{1}+b Z_{2}\right):(p, q) \mapsto\left(e^{i a} p, e^{i b} q\right)
$$

for all $a, b \in \mathbb{R}, p, q \in \mathbb{C}^{m}$.

(ii) Let $\left(B, g_{0}\right)$, resp. $\left(S, g_{0}\right)$, be the standard unit ball of dimension $4 m$, resp. the standard unit sphere of dimension $4 m-1$, in $\mathbb{R}^{4 m} \cong \mathbb{C}^{m} \oplus \mathbb{C}^{m}$. Given any $\mathfrak{z}_{\text {-valued }}$ 1-form $\lambda$ on $\mathbb{C}^{m} \oplus \mathbb{C}^{m}$ which is admissible with respect to the action of $T$ defined above, we also denote by $\lambda$ the restriction, resp. the pullback, to $B$, resp. $S$. Then $\lambda$ is admissible with respect to the induced action of $T$ on $B$ and $S$, and thus defines a corresponding metric $g_{\lambda}$ on $B$, resp. $S$. 


\subsection{Definition.}

(i) A $\mathfrak{z}$-valued 1-form $\nu$ on $\mathbb{C}^{m} \cong \mathbb{R}^{2 m}$ is called Hopf admissible if it is admissible, in the sense of 1.4 , with respect to the Hopf action of the 1-dimensional Torus $S^{1}$ on $\mathbb{C}^{m}$.

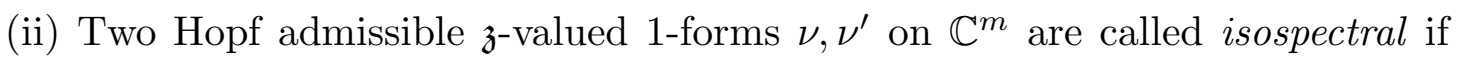
for each $\mu \in \mathcal{L}^{*}$ there exists $A_{\mu} \in \mathrm{SU}(m)$ such that $\mu \circ \nu=A_{\mu}^{*}\left(\mu \circ \nu^{\prime}\right)$.

4.9 Theorem. Let $M$ denote either the ball $B$ or the sphere $S$ in $\mathbb{C}^{m} \oplus \mathbb{C}^{m} \cong \mathbb{R}^{4 m}$. Let $\nu$ and $\nu^{\prime}$ be Hopf admissible $\mathfrak{z}$-valued 1 -forms on $\mathbb{C}^{m}$, isospectral in the sense of Definition 4.8. Write $\nu=\left(\nu_{1}, \nu_{2}\right)$ and $\nu^{\prime}=\left(\nu_{1}^{\prime}, \nu_{2}^{\prime}\right)$ with respect to the coordinates on $\mathfrak{z}=\mathbb{R}^{2}$ defined by the basis $\left\{Z_{1}, Z_{2}\right\}$. Assume that there exist $A, A^{\prime} \in \mathrm{SU}(m)$ such that $A^{*} \nu_{1}=\nu_{2}, A^{*} \nu_{2}=\nu_{1}$, and similarly for $A^{\prime}$ with respect to $\nu^{\prime}$. Define a $\mathfrak{z}$-valued 1-form $\lambda$ on $\mathbb{C}^{m} \oplus \mathbb{C}^{m}$ by

$$
\lambda_{(p, q)}(X, Y):=\nu_{p}(X)+\nu_{q}^{\prime}(Y) .
$$

for all $(p, q) \in \mathbb{C}^{m} \oplus \mathbb{C}^{m}$. Let $\psi: \mathbb{C}^{m} \cong \mathbb{R}^{2 m} \rightarrow \mathbb{R}$ be a smooth radial function and define $\varphi_{1}, \varphi_{2}: \mathbb{C}^{m} \oplus \mathbb{C}^{m} \rightarrow \mathbb{R}$ by $\varphi_{i}\left(p_{1}, p_{2}\right):=\psi\left(p_{i}\right)$ for $i=1,2$. Then

(i) $\varphi_{1}$ and $\varphi_{2}$ are isospectral potentials on $\left(M, g_{\lambda}\right)$.

(ii) If $\psi$ is strictly positive, then $\varphi_{1} g_{\lambda}$ and $\varphi_{2} g_{\lambda}$ are isospectral conformally equivalent Riemannian metrics on $M$.

Proof. The proof is very similar to that of Theorem 4.3, slightly complicated by the fact that switching the components of $\mathbb{C}^{m} \oplus \mathbb{C}^{m}$ is no longer a $T$-equivariant map here. Define a second $\mathfrak{z}$-valued 1 -form $\lambda^{\prime}$ on $\mathbb{C}^{m} \oplus \mathbb{C}^{m}$ by

$$
\lambda_{(p, q)}^{\prime}(X, Y):=\nu_{p}^{\prime}(X)+\nu_{q}(Y) .
$$

Let $g_{\lambda^{\prime}}$ be the associated Riemannian metric on $M$. Given any $\mu \in \mathcal{L}^{*}$ and the associated $A_{\mu}$ as in Definition 4.8(ii), the map

$$
F_{\mu}: \mathbb{C}^{m} \oplus \mathbb{C}^{m} \ni(p, q) \mapsto\left(A_{\mu} p, A_{\mu}^{-1} q\right) \in \mathbb{C}^{m} \oplus \mathbb{C}^{m}
$$

restricts to an isometry of $\left(M, g_{0}\right)$ satisfying $\mu \circ \lambda=F_{\mu}^{*}\left(\mu \circ \lambda^{\prime}\right)$; note that $F_{\mu}$ is $T$-equivariant since each $A_{\mu} \in \mathrm{SU}(m)$ commutes with multiplication by complex scalars. Moreover, the map

$$
\tau: \mathbb{C}^{m} \oplus \mathbb{C}^{m} \ni(p, q) \mapsto\left(A^{\prime} q, A p\right) \in \mathbb{C}^{m} \oplus \mathbb{C}^{m}
$$

restricts to a $T$-preserving isometry of $\left(M, g_{0}\right)$. The corresponding linear automorphism $\Psi_{\tau}$ of $\mathfrak{z}$ interchanges the basis vectors $Z_{1}$ and $Z_{2}$. By the conditions on $A$ and $A^{\prime}$, it is easy to check that $\tau$ satisfies $\Psi_{\tau} \circ \lambda=\tau^{*} \lambda^{\prime}$.

We let $\varphi_{1}$ play the role of $\varphi$ in the notation of 1.5 and 1.6, and observe that $\varphi_{2}=\tau^{*} \varphi_{1}$ because $\psi$ is radial. By the same reason, $\varphi_{1}$ is invariant under each $F_{\mu}$. Thus Theorem 4.9 follows from Theorem 1.5 and Corollary 1.6. 


\subsection{Example.}

We fix a realization of the Hopf projection $P: S^{3} \rightarrow S_{1 / 2}^{2} \subset \mathbb{R}^{3}$ in coordinates, say

$$
P:(\alpha, \beta, \gamma, \delta) \mapsto\left(\frac{1}{2}\left(\alpha^{2}+\beta^{2}-\gamma^{2}-\delta^{2}\right), \alpha \gamma+\beta \delta, \alpha \delta-\beta \gamma\right)
$$

We extend $P$ to a smooth map from $\mathbb{R}^{4} \cong \mathbb{C}^{2}$ to $\mathbb{R}^{3}$, defined by the same formula (note that $P$ will map $S_{a}^{3}$ to $S_{a^{2} / 2}^{2}$ for each radius $a \geq 0$ ).

With each linear map $c: \mathfrak{z} \cong \mathbb{R}^{2} \rightarrow \operatorname{Sym}_{0}\left(\mathbb{R}^{3}\right)$ we associate a $\mathfrak{z}$-valued 1 -form $\nu=\left(\nu_{1}, \nu_{2}\right)$ on $\mathbb{C}^{2}$ by letting

$$
\nu_{k}\left(X_{p}\right)=\left\langle c\left(Z_{k}\right) P(p) \times P(p), P_{* p}(X)\right\rangle
$$

for $k=1,2$ and all $X \in T_{p} \mathbb{C}^{2}$.

Consider the specific pair of linear maps $c, c^{\prime}: \mathbb{R}^{2} \rightarrow \operatorname{Sym}_{0}\left(\mathbb{R}^{3}\right)$ from Example 4.6. In addition to the three properties (isospectrality, inequivalence, genericity) stated there, we observe that

(I) There exist $E, E^{\prime} \in \mathrm{SO}(3)$ satisfying $E c\left(Z_{1}\right) E^{-1}=c\left(Z_{2}\right), E c\left(Z_{2}\right) E^{-1}=$ $c\left(Z_{1}\right)$, and similarly for $E^{\prime}$ with respect to $c^{\prime}$.

In fact, the elements $E, E^{\prime}$ of $\mathrm{SO}(3)$ given by

$$
E=\left(\begin{array}{ccc}
1 / 2 & -1 / \sqrt{2} & 1 / 2 \\
-1 / \sqrt{2} & 0 & 1 / \sqrt{2} \\
1 / 2 & 1 / \sqrt{2} & 1 / 2
\end{array}\right), \quad E^{\prime}=\left(\begin{array}{ccc}
-1 / \sqrt{2} & 0 & 1 / \sqrt{2} \\
0 & -1 & 0 \\
1 / \sqrt{2} & 0 & 1 / \sqrt{2}
\end{array}\right)
$$

satisfy $E^{2}=E^{\prime 2}=\mathrm{Id}$ and conjugate $c\left(Z_{1}\right)$ to $c\left(Z_{2}\right)$, respectively $c^{\prime}\left(Z_{1}\right)$ to $c^{\prime}\left(Z_{2}\right)$.

Again, condition 1.) on $c, c^{\prime}$ from Example 4.6 implies isospectrality of the associated Hopf admissible $\mathbb{R}^{2}$-valued 1 -forms $\nu, \nu^{\prime}$ on $\mathbb{C}^{2}$. In fact, for any given $\mu \in \mathcal{L}^{*} \subset \mathfrak{z}^{*}$ let $Z \in \mathfrak{z}$ be the dual vector (via the chosen basis). Choose $E_{Z}$ as in condition 1.), and choose $A_{Z} \in \mathrm{SU}(2) \subset \mathrm{SO}(4)$ such that $P \circ A_{Z}=E_{Z} \circ P$. Then it is straightforward to check that $A_{\mu}:=A_{Z}$ satisfies $\mu \circ \nu^{\prime}=A_{\mu}^{*}(\mu \circ \nu)$.

Moreover, condition (I) implies existence of elements $A, A^{\prime} \in \mathrm{SU}(2)$ as required in the conditions of Theorem 4.9: Just choose $A, A^{\prime}$ such that $P \circ A=E \circ P$, $P \circ A^{\prime}=E^{\prime} \circ P$. (Note that $A, A^{\prime}$ are both unique up to sign and will satisfy $A^{2}=A^{\prime 2}=-\mathrm{Id}=e^{i \pi} \mathrm{Id}$.)

Thus we are able to apply Theorem 4.9 and obtain pairs of isospectral potentials and pairs of conformally equivalent isospectral metrics on $S=S^{7} \subset \mathbb{C}^{2} \oplus \mathbb{C}^{2}$ and on $B=B^{8}$. In 5.3 we will show nontriviality of these examples for every choice of a positive smooth radial function $\psi: \mathbb{R}^{4} \cong \mathbb{C}^{2} \rightarrow \mathbb{R}_{+}$which is strictly monotonously increasing with the radius.

4.11 Remark. The choice of the metrics $g_{\lambda}$ to which our isospectral metrics are conformal, resp. which admit the pairs of isospectral potentials constructed above, 
allows an interesting modification. In each of our examples of this section $(4.4,4.6$, 4.10) one can actually modify $g_{\lambda}$ in such a way that it becomes equal to the standard metric $g_{0}$ outside certain subsets of arbitrarily small volume. The idea, first used in [Sch3] for a similar aim, is to exploit the following obvious fact:

If $\lambda, \lambda^{\prime}$ are $\mathfrak{z}$-valued admissible forms on $M$ which satisfy condition $(*)$ of Theorem 1.5, then so do $f \lambda, f \lambda^{\prime}$, where $f \in C^{\infty}(M)$ is any function which is invariant under $T$ and under each $F_{\mu}$ occurring in (*).

In particular, the statements $1.5(\mathrm{i})$ and $1.6(\mathrm{i})$ will still hold with $\lambda, \lambda^{\prime}$ replaced by $f \lambda, f \lambda^{\prime}$, and so will 1.5(ii) and 1.6(ii), provided that $f$ is invariant under $\tau$, too. In Theorems 4.3 and 4.9, which were specializations of $1.5 / 1.6$, we can therefore, without affecting any of the isospectrality statements, replace $\lambda$ by $f \lambda$, where $f$ is the restriction to $M$ of

- (for Theorem 4.3, hence the Examples 4.4 and 4.6:) any smooth function on $\mathbb{R}^{m} \oplus \mathbb{R}^{m} \oplus \mathbb{C}^{r}$ which is of the form $(p, q, u) \mapsto \alpha(|p|,|q|,|u|)$ with $\alpha(r, s, t)=$ $\alpha(s, r, t)$ for all $(r, s, t)$;

- (for Theorem 4.9, hence Example 4.10:) any smooth function on $\mathbb{C}^{m} \oplus \mathbb{C}^{m}$ which is of the form $(p, q) \mapsto \alpha(|p|,|q|)$ with $\alpha(a, b)=\alpha(b, a)$ for all $(a, b)$.

In particular, we may in each case choose $\alpha$ to have as small support (in the parameter set relevant for $M$ ) as we like. Then the support of $f$ in $M$ will have small volume with respect to the standard metric $g_{0}$ and hence also with respect to $g_{f \lambda}$ (recall from 1.4(iii) that $d$ vol $_{g_{0}}=$ dvol $_{g_{f \lambda}}$ ).

In 5.4, we will show that the corresponding nonisometry statements will remain true, provided that $f$ does not vanish identically on $M$; only in the case of Example 4.10 and $M=B^{8}$, we will need an additional technical assumption: Namely, that the support of $\alpha$ nontrivially intersects a certain open set $\mathcal{U} \subset\left\{(a, b) \in B_{1}(0)\right.$ $a>b>0\}$ (whose definition will actually depend on the radial function $\psi$ used for constructing the potentials).

\section{$\S 5$ Non-Triviality of the EXAmples}

\subsection{Nonisometry proof for the examples from 3.7.}

We consider the construction of metrics and potentials on Lie groups given in Example 3.7(i-iii). We first prove that for a special choice of the function $\varphi$ and with a very mild genericity condition on $j$ (condition 5.1.2(a) below), the isospectral potentials $\varphi$ and $\psi:=\tau^{*} \varphi$ on $\left(G, g_{\lambda}\right)$ are not congruent if $j_{1}$ and $j_{2}$ are inequivalent (Theorem 5.1.8). We then show under an additional genericity hypothesis on $j=$ $\left(j_{1}, j_{2}\right)$ (condition 5.1.2(b)) that the isospectral conformally equivalent metrics $\varphi g_{\lambda}$ and $\psi g_{\lambda}$ are not isometric (Theorem 5.1.13). For notational simplicity, we will focus throughout on the two cases $G=\mathrm{SO}(2 m+2 r)$ and $G=\mathrm{SU}(2 m+r+1)$. With minor adjustments to the proofs in the case of $\mathrm{SO}(2 m+2 r)$, one obtains the same results for $G=\operatorname{Spin}(2 m+2 r)$. 


\subsubsection{The function $\varphi$.}

For any matrix $A \in M(m, m, \mathbb{C})$ and any column vector $V \in \mathbb{C}^{m}$ we write

$$
d(A, V):=\operatorname{det}\left(V, A V, A^{2} V, \ldots, A^{m-1} V\right) .
$$

Note that $d$ is invariant under the action of $\mathrm{SL}_{\mathbb{C}}(m)$ on $M(m, m, \mathbb{C}) \times \mathbb{C}^{m}$ given by $B(A, V)=\left(B A B^{-1}, B V\right)$ for $B \in \mathrm{SL}_{\mathbb{C}}(m)$. It is easy to see that if $A$ has pairwise different eigenvalues $\lambda_{1}, \ldots, \lambda_{m}$, then $d(A, V)$ is the Vandermonde determinant $\left|\left(\lambda_{k}^{\ell}\right)\right|_{\substack{1 \leq k \leq m \\ 0 \leq \ell \leq m-1}}=\prod_{i<j}\left(\lambda_{j}-\lambda_{i}\right)$, multiplied by the product of the coefficients of $V$ with respect to an eigenbasis of determinant one of $A$. If $A$ is diagonizable and has at least one multiple eigenvalue, then $d(A, V)=0$ for all $V$.

For $A \in M(m, m, \mathbb{C})$ and $C \in M(m, \ell, \mathbb{C})$ we let

$$
d(A, C):=\prod_{k=1, \ldots, \ell} d\left(A, C_{k}\right)
$$

where $C_{1}, \ldots, C_{\ell}$ denote the column vectors of $C$.

We write matrices in $G=\mathrm{SO}(2 m+2 r)$ or $G=\mathrm{SU}(2 m+r+1)$ as

$$
X=\left(\begin{array}{lll}
A & B & C \\
D & E & F \\
H & J & L
\end{array}\right),
$$

where $A, B, D, E$ are $m \times m$-matrices and $L$ is a $2 r \times 2 r$-matrix or an $(r+1) \times$ $(r+1)$-matrix, respectively. Let $c_{1}>c_{2}>0$. In case $G=\mathrm{SO}(2 m+2 r)$, we define $\varphi: G \rightarrow \mathbb{R}_{+}$by

$$
\varphi(X):=\exp \left(c_{1} \operatorname{det} A+c_{2} \operatorname{det} E\right) .
$$

In case $G=\mathrm{SU}(2 m+r+1)$, we let

$$
\varphi(X):=\exp \left(c_{1} \operatorname{Re} \operatorname{det} A+c_{2} \operatorname{Re} \operatorname{det} E-(\operatorname{Re} d(A, C))^{2}-(\operatorname{Re} d(E, F))^{2}\right),
$$

where Re denotes the real part.

\subsubsection{Genericity conditions.}

(a) The kernel of the map $j=\left(j_{1}, j_{2}\right): \mathfrak{z} \rightarrow \mathfrak{h}$ is trivial, where $\mathfrak{h}=\mathfrak{k} \oplus \mathfrak{k}$.

(b) The image of $j$ has trivial centralizer in $\mathfrak{h}$.

5.1.3 Notation. For any subgroup $U$ of $G$, denote by $U_{L}$, respectively $U_{R}$, the group of all left, respectively right, translations of $G$ by elements of $U$. Moreover, denote by $\operatorname{Inn}_{G}(U)$ the subgroup of the group of inner automorphisms of $G$ consisting of conjugations by elements of $U$. 
5.1.4 Lemma [OT]. Let $G$ be a connected simple compact Lie group with a left invariant Riemannian metric $g$. Then:

(i) The full group of isometries of $(G, g)$ is the semi-direct product of $G_{L}$ with the group of all automorphims of $G$ that preserve $g$.

(ii) Let $W=\left\{a \in G \mid R_{a}\right.$ preserves $\left.g\right\}$ and let $Z(G)$ be the (finite) center of $G$. Then the identity component $\operatorname{Isom}_{0}(G, g)$ of the full isometry group is given by $\left(G_{L} \times W_{R}\right) / Z(G)$, where $Z(G)$ is embedded by $a \mapsto L_{a} \circ R_{a}^{-1}$.

Note that $a \in W$ if and only if the associated inner automorphism $I_{a}$ of $G$ is an isometry of $(G, g)$.

5.1.5 Notation. Let $P$ be the subgroup of $G$ given by those matrices of the form

$$
\left(\begin{array}{ccc}
\operatorname{Id}_{m} & 0 & 0 \\
0 & \operatorname{Id}_{m} & 0 \\
0 & 0 & *
\end{array}\right) .
$$

Thus $P$ is isomorphic to $\mathrm{SO}(2 r)$, respectively $\mathrm{SU}(r+1)$, in case $G=\mathrm{SO}(2 m+2 r)$, respectively $G=\mathrm{SU}(2 m+r+1)$. Let $Q=H \times P$. Recall that $H$ is the subgroup $\mathrm{SO}(m) \times \mathrm{SO}(m)$, respectively $\mathrm{SU}(m) \times \mathrm{SU}(m)$, of $G$, embedded in the upper left corner, and that $T$ is the standard maximal torus in $P$.

5.1.6 Lemma. Let $\varphi$ be defined as in 5.1.1. Let $\mathcal{G}$ denote the subgroup of $G_{L} \times G_{R}$ consisting of those elements which leave $\varphi$ invariant, and let $\mathcal{G}_{0}$ denote the connected component of the identity in $\mathcal{G}$. Then:

(i) If $L_{a} R_{b} \in \mathcal{G}$ then $a \cdot b \in Q$ and $a, b$ are elements of $\mathrm{S}(\mathrm{O}(m) \times \mathrm{O}(m) \times \mathrm{O}(2 r))$, respectively of $\mathrm{S}(\mathrm{U}(m) \times \mathrm{U}(m) \times \mathrm{U}(r+1))$. In particular, $I_{a}$ and $I_{b}$ leave the subgroups $H$ and $P$ of $G$ invariant.

(ii) $\mathcal{G}_{0}=Q_{L} \times Q_{R}$ in case $G=\mathrm{SO}(2 m+2 r)$, and

$\mathcal{G}_{0}=\operatorname{Inn}_{G}(H) \times P_{L} \times T_{R}$ in case $G=\mathrm{SU}(2 m+r+1)$.

Proof. (i) It is obvious from the definition of $\varphi$ that it assumes its maximum value precisely on $Q \subset G$. By determining first which elements of $G_{L} \times G_{R}$ preserve $Q$, and then ruling out those of these candidates which obviously do not preserve $\varphi$ (taking into particular account that $c_{1}>c_{2}>0$ ) it is not hard to see that $a$ and $b$ must be of the asserted form. Moreover, $a Q b=Q$ implies $a \cdot b \in Q$.

(ii) If $G=\mathrm{SO}(2 m+2 r)$ then (i) implies $\mathcal{G}_{0} \subset Q_{L} \times Q_{R}$. Since $Q_{L} \times Q_{R}$ does leave $\varphi$ (as defined for this $G$ ) invariant, the statement follows.

Now let $G=\mathrm{SU}(2 m+r+1)$. In this case, (i) says that $\mathcal{G}$, and hence $\mathcal{G}_{0}$, is contained in

$$
Q_{L} \times Q_{R} \times \operatorname{Inn}_{G}(D)=H_{L} \times H_{R} \times P_{L} \times P_{R} \times \operatorname{Inn}_{G}(D),
$$


where $D$ is the subgroup of $G$ consisting of the matrices $\left(\begin{array}{ccc}\alpha \operatorname{Id}_{m} & & \\ & \beta \operatorname{Id}_{m} & \\ & & \\ & & \\ & & \\ & & \\ \operatorname{Id}_{r+1}\end{array}\right) \in G$ with $\alpha, \beta, \gamma \in e^{i \mathbb{R}}$. It is clear from the definition of $\varphi$ and the comments in 5.1.1 that $\operatorname{Inn}_{G}(H) \times P_{L} \times T_{R}$ does preserve $\varphi$, hence is contained in $\mathcal{G}_{0}$. We have to show that it is in fact equal to $\mathcal{G}_{0}$. We claim that any 1-parameter family in $H_{L} \times P_{R} \times$ $\operatorname{Inn}_{G}(D)$ which leaves $\varphi$ invariant is actually contained in $T_{R}$; this will obviously imply the statement. Let $h(t), p(t), d(t)$ be 1 -parameter families in $H, P$, and $D$, respectively, with the property that $L_{h(t)} R_{p(t)} I_{d(t)}$ leaves $\varphi$ invariant. Write $h(t)=$ $\left(\begin{array}{ccc}k_{1}(t) & & \\ & k_{2}(t) & \\ & & \text { Id }\end{array}\right)$, where $k_{1}(t), k_{2}(t)$ are 1-parameter families in $\mathrm{SU}(m)$, and write $d(t)$ as above with 1-parameter families $\alpha(t), \beta(t), \gamma(t)$ in $e^{i \mathbb{R}}$. Identify $p(t)$ with the corresponding 1-parameter family in $\mathrm{SU}(r+1)$. Note that the first two summands in the exponent of $\varphi$ are invariant under $L_{h(t)} R_{p(t)} I_{d(t)}$; consequently, this family must also leave the function $\psi: G \ni X \mapsto(\operatorname{Re} d(A, C))^{2}+(\operatorname{Re} d(E, F))^{2} \in \mathbb{R}$ invariant, where $X$ is written as in 5.1.1.

We first show that the family $h(t)$ is trivial. Assume the contrary. Then $k_{1}(t)$ or $k_{2}(t)$ is nontrivial; without loss of generality we assume $k_{1}(t)$ is nontrivial. Choose $t_{0}$ such that $k_{1}\left(t_{0}\right)$ has at least two different eigenvalues. Choose $A^{\prime} \in \mathrm{SU}(m)$ such that $A^{\prime}$ has less than $m$ different eigenvalues, but $k_{1}\left(t_{0}\right)^{-1} A^{\prime}$ has $m$ pairwise different eigenvalues. Choose $0<c<1$. It is easy to see that there exist elements $X$ of $G$, written as in 5.1.1, with $A=c k_{1}\left(t_{0}\right)^{-1} A^{\prime}$ and $F=0$. It is also not hard to show that the condition that no column vector of $C$ be orthogonal to any of the eigenvectors of $A$ - equivalently, $d(A, C) \neq 0-$ is a generic condition on this subset of $G$, and that so is the condition $\operatorname{Re} d(A, C) \neq 0$. Choose $X \in G$ with these properties. Then $\psi(X)=(\operatorname{Re} d(A, C))^{2}+0 \neq 0$, but $\psi\left(L_{h\left(t_{0}\right)} R_{p\left(t_{0}\right)} I_{d\left(t_{0}\right)} X\right)=$ $\left(\operatorname{Re} d\left(c A^{\prime}, k_{1}\left(t_{0}\right) C p\left(t_{0}\right) \alpha\left(t_{0}\right) \gamma\left(t_{0}\right)^{-1}\right)^{2}+0=0\right.$ since $A^{\prime}$ has less than $m$ different eigenvalues (recall the comments in 5.1.1). This is a contradiction; so the family $h(t)$ must indeed be trivial.

Next we attack $p(t)$. Assume that the family $p(t)$ were not contained in the maximal torus $T$ consisting of the diagonal elements in $P$. Let $k$ be a diagonal matrix in $\mathrm{SU}(m)$ with $m$ different eigenvalues, and set $A:=c k$ for some $c$ with $0<c<1$. Choose $t_{0}$ such that some column vector $p_{i}\left(t_{0}\right)$ of $p\left(t_{0}\right)$ has at least two nonzero entries. It is easy to see that there exist elements $X$ of $G$, written as in 5.1.1, with the given $A=c k$ and with $F=0$, such that some row vector of $C$ is orthogonal to $p_{i}\left(t_{0}\right)$. It is also not hard to show that the condition that $C$ have only nonvanishing entries - equivalently: $d(A, C) \neq 0$ - is a generic condition on this subset of $G$ (recall the choice of $p_{i}\left(t_{0}\right)$ ), and that so is the condition $\operatorname{Re} d(A, C) \neq 0$. Choose $X \in G$ with these properties. Then $\psi(X)=(\operatorname{Re} d(A, C))^{2} \neq 0$, but $\psi\left(L_{h\left(t_{0}\right)} R_{p\left(t_{0}\right)} I_{d\left(t_{0}\right)} X\right)=\left(\operatorname{Re} d\left(A, C p\left(t_{0}\right) \alpha\left(t_{0}\right) \gamma\left(t_{0}\right)^{-1}\right)^{2}=0\right.$ since $C p_{i}\left(t_{0}\right)$ has a zero entry and thus $d\left(A, C p_{i}\left(t_{0}\right)\right)=0$ (recall that $A$ is diagonal). This is a contradiction, so the family $p(t)$ must be contained in $T$. 
Finally, for every $X \in G$ with $F=0$ we have that $\psi\left(R_{p(t)} I_{d(t)} X\right)$ is equal to $\left(\operatorname{Re} d\left(A, C p(t) \alpha(t) \gamma(t)^{-1}\right)^{2}=\left(\operatorname{Re}\left(\left(\alpha(t) \gamma(t)^{-1}\right)^{m(r+1)} d(A, C)\right)^{2}\right.\right.$ since $p(t) \in T$. The fact that for every given such $X$ this expression must be constant in $t$ implies $\alpha(t)=\gamma(t)$ for all $t$. Analogously we obtain $\beta(t)=\gamma(t)$ for all $t$, hence $d(t)=$ $\alpha(t) \operatorname{Id}_{2 m+r+1}$. But $d(t) \in \mathrm{SU}(2 m+r+1)$; hence the family $d(t)$ is trivial.

5.1.7 Lemma. Assume that $j$ satisfies the genericity condition 5.1.2(a). Let $\Phi$ be any automorphism of $Q$ which preserves the subgroups $H$ and $P$ and which is an isometry of the metric $g_{\lambda}$. Then $\Phi$ preserves $T$, and $\left(\left.\Phi_{*}^{-1}\right|_{\mathfrak{h}},\left.\Phi_{*}\right|_{\mathfrak{z}}\right)$ is a selfequivalence of $j$ in the sense of Definition 3.1.

Proof. For $X \in \mathfrak{h}$ and $U \in \mathfrak{p}$ we have $g_{\lambda}(X, U)=g_{0}(\lambda(X), U)$ since $\left.\lambda\right|_{\mathfrak{p}}=0$ and since $\mathfrak{h}$ is $g_{0}$-orthogonal to $\mathfrak{p}$. From the facts that $g_{\lambda}(X, U)=g_{\lambda}\left(\Phi_{*}(X), \Phi_{*}(U)\right)$ and that $\Phi_{*}$ preserves $\mathfrak{h}$ and $\mathfrak{p}$, it follows that

$$
g_{0}(\lambda(X), U)=g_{0}\left(\lambda\left(\Phi_{*} X\right), \Phi_{*} U\right) .
$$

Using the genericity condition 5.1.2(a), we may conclude that $\Phi_{*}\left(\mathfrak{p} \cap \mathfrak{z}^{\perp}\right) \subset \mathfrak{p} \cap \mathfrak{z}^{\perp}$ and thus $\Phi_{*}(\mathfrak{z})=\mathfrak{z}$. Letting $U \in \mathfrak{z}$ in the displayed equation above, we then have

$$
g_{0}(X, j(U))=g_{0}\left(\Phi_{*} X, j\left(\Phi_{*} U\right)\right)=g_{0}\left(X, \Phi_{*}^{-1}\left(j\left(\Phi_{*} U\right)\right)\right),
$$

and the lemma follows.

5.1.8 Theorem. We use the notation of Example 3.7. Let $\varphi$ be the function given in 5.1.1 and let $\psi=\tau^{*} \varphi$. Assume that $j_{1}$ is not equivalent to $j_{2}$ and that $j$ satisfies the genericity condition 5.1.2(a). Then $\psi$ is not congruent to $\varphi$ under an isometry of $\left(G, g_{\lambda}\right)$.

Thus by Example 3.7, $\varphi$ and $\psi$ are non-congruent isospectral potentials on $\left(G, g_{\lambda}\right)$.

Proof. Suppose $F$ is an isometry of $\left(G, g_{\lambda}\right)$ such that $F^{*} \psi=\varphi$. By Lemma 5.1.4, $F \in G_{L} \rtimes \operatorname{Aut}(G)$. Recall that $\tau=I_{c}$, where

$$
c=\left(\begin{array}{ccc}
0 & \text { Id } & 0 \\
\text { Id } & 0 & 0 \\
0 & 0 & \text { Id }
\end{array}\right) .
$$

Thus $I_{c}^{*} \psi=\varphi$, so $\varphi$ is invariant under $I_{c^{-1}} \circ F$. Let $\sigma$ be the outer automorphism of $G$ given (in case $G=\mathrm{SO}(2 m+2 r)$ ) by conjugation by a diagonal matrix of determinant -1 all of whose diagonal entries lie in $\{ \pm 1\}$, or (in case $G=\mathrm{SU}(2 m+r+1)$ ) by the map $A \mapsto \bar{A}$. Observe that $\sigma$ leaves $\varphi$ invariant. Since for both choices of $G$, $\operatorname{Inn}(G)$ has index two in $\operatorname{Aut}(G), I_{c}^{-1} \circ F$ may be written either as $\sigma \circ I_{a} \circ L_{b}$ or else as $I_{a} \circ L_{b}$ for some $a, b \in G$. In either case $\left(I_{a} \circ L_{b}\right)^{*} \varphi=\varphi$, so Lemma 5.1.6(i) implies that $I_{a}$ and $I_{b}$ preserve $H$ and $P$. Consequently, the automorphism $\Phi:=F \circ L_{b}^{-1}$ 
(which is either $I_{c a}$ or $\left.I_{c} \circ \sigma \circ I_{a}\right)$ is an isometry of $\left(G, g_{\lambda}\right)$ which preserves both $H$ and $P$ and interchanges the two factors of $H$. By Lemma 5.1.7, we see that $\left(\left.\Phi_{*}^{-1}\right|_{\mathfrak{h}},\left.\Phi_{*}\right|_{\mathfrak{z}}\right)$ is a self-equivalence of $j$ which intertwines $j_{1}$ and $j_{2}$, contradicting the fact that $j_{1}$ and $j_{2}$ are not equivalent.

We now prepare for the proof that the conformally equivalent metrics in Example 3.7 are not isometric for our given choice of $\varphi$ and with the genericity hypotheses 5.1.2.

5.1.9 Proposition. Suppose that $(M, g)$ is a compact Riemannian manifold admitting a free action by isometries by a torus $T$ and that the orbits all have the same volume (i.e., they are totally geodesic). Let $\varphi$ and $\psi$ be positive smooth $T$-invariant functions on $M$. Let $S$ be a non-trivial subtorus of $T$ and let $F:(M, \varphi g) \rightarrow$ $(M, \psi g)$ be an isometry that carries $S$-orbits to $S$-orbits. Then $F^{*} \psi=\varphi$ and $F \in \operatorname{Isom}(M, g)$. In particular (letting $\psi=\varphi)$, the normalizer of $S$ in $\operatorname{Isom}(M, \varphi g)$ is contained in $\left\{F \in \operatorname{Isom}(M, g) \mid F^{*} \varphi=\varphi\right\}$.

Proof. The second statement is immediate from the first. To prove that $F^{*} \psi=\varphi$, first note that all $S$-orbits have the same volume relative to the metric $g$; in fact, they are totally geodesic. Moreover, since $\varphi$ and $\psi$ are $S$-invariant, they are constant on each orbit. Since the isometry $F$ preserves volumes, it must intertwine the values of $\varphi$ and $\psi$ on each orbit. Hence $\psi \circ F=\varphi$. Consequently, $F$ is an isometry of $(M, g)$.

5.1.10 Lemma. Let 5.1.2(a,b) be satisfied. Denote by $C$ the connected component of the identity in $\left\{F \in \operatorname{Isom}\left(G, g_{\lambda}\right) \mid F^{*} \varphi=\varphi\right\}$. Then $C=Q_{L} \times T_{R}$ in case $G=\mathrm{SO}(2 m+2 r)$, and $C=P_{L} \times T_{R}$ in case $G=\mathrm{SU}(2 m+r+1)$, where $Q$ and $P$ are as in Notation 5.1.5.

Proof. If $G=\mathrm{SO}(2 m+2 r)$ then by Lemmas 5.1.4 and 5.1.6(ii) and the fact that $\varphi$ and $g_{\lambda}$ are invariant under $Q_{L} \times T_{R}$, we have $Q_{L} \times T_{R} \subset C \subset Q_{L} \times Q_{R}$. If $G=\mathrm{SU}(2 m+r+1)$ then the same lemmas and the fact that $\varphi$ is invariant under $P_{L} \times T_{R}$ imply $P_{L} \times T_{R} \subset C \subset \operatorname{Inn}_{G}(H) \times P_{L} \times T_{R}$. By Lemma 5.1.7 and the fact that the group of automorphisms of the torus $T$ is discrete, the only oneparameter subgroups of $\operatorname{Inn}_{G}(Q)$ which lie in $\operatorname{Isom}\left(g_{\lambda}\right)$ (consequently also: the only such subgroups of $\left.\operatorname{Inn}_{G}(H)\right)$ are those which restrict to the identity on $\mathfrak{z}$ and also on $j(\mathfrak{z})$. Since the centralizer of $j(\mathfrak{z})$ in $\mathfrak{h}$ is trivial by condition 5.1.2(b), and since the centralizer of $\mathfrak{z}$ in $\mathfrak{p}$ is $\mathfrak{z}$, these one-parameter subgroups must lie in $\operatorname{Inn}_{G}(T)$. The corollary follows.

5.1.11 Lemma. Let $U$ be a compact Lie group and $T$ a torus in $U$ of dimension greater than one. Suppose that every subtorus of $T$ of positive dimension has the same centralizer in $U$. Then $T$ lies in the center of $U$ (and thus its centralizer is $U)$. 
Proof. Let $\mathfrak{u}$ denote the Lie algebra of $U$ and $\mathfrak{t} \subset \mathfrak{u}$ the Lie algebra of $T$. Consider the simultaneous root space decomposition of the complexified space $\mathfrak{u}^{\mathbb{C}}$ with respect to the operators ad $(X), X \in \mathfrak{t}$. If $Y=Y_{1}+i Y_{2}$ (with $Y_{1}, Y_{2} \in \mathfrak{u}$ ) is a root vector, say $\operatorname{ad}(X)(Y)=i \alpha(X) Y$ for all $X \in \mathfrak{u}$, where $\alpha \in \mathfrak{t}^{*}$, then either $\alpha \equiv 0$ or else $\operatorname{ker}(\alpha)$ is a subspace $\mathfrak{s}$ of $\mathfrak{t}$ of codimension one. In the latter case the centralizer of the associated subtorus $S$ of $T$ contains $Y$ and thus properly contains the centralizer of $T$, contradicting our hypothesis. Thus $\alpha \equiv 0$ for every root $\alpha$. Hence $\mathfrak{t}$ is central in $\mathfrak{u}$ and $T$ is central in $U$.

5.1.12 Proposition. If the genericity conditions 5.1.2(a,b) are satisfied, then the identity component of the center of $\operatorname{Isom}_{0}\left(G, \varphi g_{\lambda}\right)$ is equal to $T_{R}$.

Proof. By Proposition 5.1.9 and Lemma 5.1.10, the centralizer in $\operatorname{Isom}_{0}\left(G, \varphi g_{\lambda}\right)$ of any nontrivial subtorus of $T_{R}$ is given in case $G=\mathrm{SO}(2 m+2 r)$ by $Q_{L} \times T_{R}$ and in case $G=\mathrm{SU}(2 m+r+1)$ by $P_{L} \times T_{R}$. Thus by Lemma $5.1 .11, \operatorname{Isom}_{0}\left(G, g_{\lambda}\right)$ equals $Q_{L} \times T_{R}$, respectively $P_{L} \times T_{R}$. Since in either case, $T_{R}$ is the identity component of the center of this group, the proposition follows.

Of course, the proposition is also valid with $\varphi$ replaced by $\psi$.

5.1.13 Theorem. Let $\varphi$ be the function given in 5.1 .1 and let $\psi=\tau^{*} \varphi$. Assume that $j_{1}$ is not equivalent to $j_{2}$ and that $j$ satisfies the genericity conditions $5.1 .2(\mathrm{a}, \mathrm{b})$. Then the metrics $\varphi g_{\lambda}$ and $\psi g_{\lambda}$ on $G$ are not isometric.

Proof. Suppose that $F:\left(G, \varphi g_{\lambda}\right) \rightarrow\left(G, \psi g_{\lambda}\right)$ were an isometry. By Proposition 5.1.12, the resulting isomorphism from $\operatorname{Isom}\left(G, \varphi g_{\lambda}\right)$ to $\operatorname{Isom}\left(G, \psi g_{\lambda}\right)$ given by $\mu \mapsto F \circ \mu \circ F^{-1}$ carries $T_{R}$ to $T_{R}$. Hence $F$ carries $T_{R}$-orbits in $G$ to $T_{R}$-orbits. By Proposition 5.1.9, $F$ is an isometry of $g_{\lambda}$ and satisfies $F^{*} \psi=\varphi$, contradicting Theorem 5.1.8.

\subsection{Nonisometry proof for the examples from 4.4/4.6.}

We prove here the nonisometry of the conformally equivalent metrics on both the balls and the spheres in Examples 4.4/4.6. As an aside, we observe that the nonisometry of the metrics on the balls would follow immediately from that on the boundary spheres. However, we will not use this observation below but instead prove the nonisometry of the metrics on the balls directly, so that we will be able later (in 5.4) to adapt the proof to address the nonisometry of the metrics in Remark 4.11, where the boundary spheres may in fact be isometric.

In the context of Example 4.4 (resp. 4.6), suppose that the linear maps $j, j^{\prime}: \mathfrak{z}=$ $\mathbb{R}^{2} \rightarrow \mathfrak{s o}(m)$ (resp. $c, c^{\prime}: \mathbb{R}^{2} \rightarrow \operatorname{Sym}_{0}\left(\mathbb{R}^{3}\right)$ ) satisfy the conditions from Remark 4.5 (resp. those specified in 4.6); let $\nu, \nu^{\prime}$ be the associated $\mathbb{R}^{2}$-valued 1 -forms on $\mathbb{R}^{m}$ (where $m=3$ in the case of 4.6). Furthermore, assume that the positive smooth radial function $\psi$ on $\mathbb{R}^{m}$ satisfies the condition from 4.5 (resp. 4.6); writing $\psi(p)=$ $\rho(|p|)$, this means that $\left.\rho\right|_{[0,1]}$ attains its maximum precisely in 0 . 
For $M=B \subset \mathbb{R}^{m} \oplus \mathbb{R}^{m} \oplus \mathbb{C}^{2}$, or $M=S \subset \mathbb{R}^{m} \oplus \mathbb{R}^{m} \oplus \mathbb{C}^{2}$, we are to show that the metrics $\varphi_{1} g_{\lambda}$ and $\varphi_{2} g_{\lambda}$ on $M$ are nonisometric, where $\lambda$ is the $\mathbb{R}^{2}$-valued 1-form associated with $\nu \oplus \nu^{\prime}$ on $\mathbb{R}^{m} \oplus \mathbb{R}^{m}$ as in 4.1(ii), and where the $\varphi_{i}(i=1,2)$ are defined as in Theorem 4.3. By the proof of Theorem 4.3, the metric $\varphi_{2} g_{\lambda}$ is isometric to $\varphi_{1} g_{\lambda^{\prime}}$, where $\lambda^{\prime}$ is the $\mathbb{R}^{2}$-valued 1-form associated with $\nu^{\prime} \oplus \nu$. Following the strategy described in Proposition 2.2 and Remark 2.3, it thus suffices to show that the 1-forms $\lambda$ and $\lambda^{\prime}$ satisfy the conditions $(\mathrm{N})$ and $(\mathrm{G})$ in Proposition 2.2 with respect to the metric $\varphi g_{0}$ on $M$, where $\varphi:=\varphi_{1}$. Recall that $\varphi_{1}(p, q, u)=\psi(p)=$ $\rho(|p|) \in \mathbb{R}_{+}$.

We canonically identify the set $\hat{M} / T$ (see Notation 1.2 ) with

$$
B^{2 m+2} \cap Q=\left\{\left.(p, q, a, b) \in \mathbb{R}^{m} \oplus \mathbb{R}^{m} \oplus \mathbb{R} \oplus \mathbb{R}|a, b>0,| p\right|^{2}+|q|^{2}+a^{2}+b^{2}<1\right\}
$$

in case $M=B$, resp. with

$$
S^{2 m+1} \cap Q=\left\{\left.(p, q, a, b) \in \mathbb{R}^{m} \oplus \mathbb{R}^{m} \oplus \mathbb{R} \oplus \mathbb{R}|a, b>0,| p\right|^{2}+|q|^{2}+a^{2}+b^{2}=1\right\}
$$

in case $M=S$, where $Q$ denotes the open "quadrant" of $\mathbb{R}^{2 m+2}$ defined by $a, b>0$. The metric $\varphi g_{0}^{T}$ on $\hat{M} / T$ is in each case given as $\bar{\varphi} g_{0}$, where $\bar{\varphi}(p, q, a, b):=\rho(|p|)$, and where $g_{0}$ now denotes the standard metric on $\mathbb{R}^{2 m+2}$ as well.

Let $\bar{F} \in \overline{\operatorname{Aut}}_{\varphi g_{0}}^{T}(M)$.

If $M=B$ then $\bar{F}$, being an isometry of $\bar{\varphi} g_{0}$, extends to a continuous self-map, again denoted $\bar{F}$, of the closure of $B^{2 m+2} \cap Q$. Note that $\bar{F}$ must preserve the $2 m$-dimensional "edge" $B^{2 m} \times\{0\} \subset \mathbb{R}^{2 m} \oplus \mathbb{R}^{2}$ because this is the image under the projection $M \rightarrow M / T$ of those points whose $T$-orbit is just a point. Hence $\bar{F}$ must also preserve the opposite "face" $S^{2 m+1} \cap Q$. So $\bar{F}$ preserves $S^{2 m+1} \cap Q$ regardless of whether $M=B$ or $M=S$.

Note that the $T$-orbit of a point $(p, q, v, w) \in M$ with $|v|=a$ and $|w|=b$, endowed with the metric induced by $\varphi g_{0}$, is a rectangular torus with side lengths $\sqrt{\rho(|p|)} \cdot 2 \pi a$ and $\sqrt{\rho(|p|)} \cdot 2 \pi b$. Considering the ratio of the side lengths, we see that $\bar{F}$ must either preserve or switch the functions $(p, q, a, b) \mapsto a / b$ and $(p, q, a, b) \mapsto b / a$ on $\hat{M} / T$ and thus on $S^{2 m+1} \cap Q$ (by continuity in case $M=B$ ).

The length of the diagonal of the rectangular torus which is the $T$-orbit of a point $(p, q, a, b) \in \hat{M} / T$ is equal to $\ell(p, q, a, b)$, where $\ell: \mathbb{R}^{2 m+2} \ni(p, q, a, b) \mapsto$ $\sqrt{\rho(|p|)} \cdot 2 \pi \sqrt{a^{2}+b^{2}} \in \mathbb{R}$. Consequently $\bar{F}$ must preserve $\ell$, too. By our assumption on $\psi$ (hence on $\rho$ ), the restriction of $\ell$ to $S^{2 m+1} \cap Q$ attains its maximum precisely in the points $(0,0, a, b)$; hence the set of these points, too, is invariant under $\bar{F}$. Summarizing, for each vector $(a, b) \in S^{1}$ with $a, b>0$, the map $\bar{F}$ either preserves or switches the two $2 m$-dimensional hemispheres $S_{(a, b)}$ and $S_{(b, a)}$ where $S_{(a, b)}:=$ $S^{2 m+1} \cap\left\{(p, q, t a, t b) \mid p, q \in \mathbb{R}^{m}, t \in \mathbb{R}_{+}\right\}$, and accordingly $\bar{F}$ fixes, resp. switches, their poles $(0,0, a, b)$ and $(0,0, b, a)$. 
Recall that $\bar{F}$ is, at the same time, an isometry of $\bar{\varphi} g_{0}$. In particular, it is a conformal self-map of the open domain $B^{2 m+2} \cap Q \subset \mathbb{R}^{2 m+2}$ (in case $M=B$ ), resp. of the open domain $S^{2 m+1} \cap Q \subset S^{2 m+1}$ (in case $M=S$ ). By Liouville's theorem (see, e.g., [Sp], pp. 302-313), and since the dimensions of these domains are certainly greater than two, it follows that $\bar{F}$ is the restriction to $\hat{M} / T$ of a Möbius transformation $\Phi$ of $\overline{\mathbb{R}^{2 m+2}}:=\mathbb{R}^{2 m+2} \cup\{\infty\}$; that is, a composition of reflections in hyperspheres and hyperplanes. (To see this for $S^{2 m+1} \cap Q$, one has to conjugate $\bar{F}$ by the stereographical projection, which is itself the restriction of a Möbius transformation, and to use the fact that each Möbius transformation of $\overline{\mathbb{R}^{2 m+1}}$ extends to one of $\overline{\mathbb{R}^{2 m+2}}$.)

From our previous arguments we now conclude that $\Phi$, combined with the reflection in the $(a=b)$-hyperplane if necessary, preserves each of the $2 m$-dimensional hemispheres $S_{(a, b)}$ considered above and, moreover, fixes their poles $(0,0, a, b)$. By well-known properties of Möbius transformations, this implies that on each of these hemispheres, $\Phi$ is uniquely determined by its restriction to the boundary $(2 m-1)$-sphere, on which it acts as an orthogonal transformation. Since all the hemispheres $S_{(a, b)}$ have the same boundary, namely, $S^{2 m-1} \times\{0\} \subset \mathbb{R}^{2 m} \oplus \mathbb{R}^{2}$, it follows that $\left.\Phi\right|_{S^{2 m+1}}$ is of the form $(U, \sigma)$ with $U \in \mathrm{O}(2 m)$ and $\sigma \in\left\{\operatorname{Id},\left(\begin{array}{ll}0 & 1 \\ 1 & 0\end{array}\right)\right\} \subset$ $\mathrm{O}(2)$.

Recall once more that $\bar{F}$ - and hence $\Phi$ - has to preserve the function $\ell$ : $(p, q, a, b) \mapsto \sqrt{\rho(|p|)} \cdot 2 \pi \sqrt{a^{2}+b^{2}}$ on $S^{2 m+1} \cap Q$. Since our assumption on $\rho$ implies, in particular, that $\rho$ is nonconstant on $[0,1]$, it follows that $U$ is of the form $\left(A, A^{\prime}\right) \in$ $\mathrm{O}(m) \times \mathrm{O}(m)$. Thus $\left.\Phi\right|_{S^{2 m+1}}=\left(A, A^{\prime}, \sigma\right)$. If $M=B$ then $\Phi$ must moreover preserve $B^{2 m+2}$ because it extends $\bar{F}$. Summarizing, we have shown so far:

$$
\begin{gathered}
\text { Each } \bar{F} \in \overline{\operatorname{Aut}}_{\varphi g_{0}}^{T}(M) \text { is the restriction to } \hat{M} / T \subset \mathbb{R}^{2 m+2} \text { of } \\
\text { some map }\left(A, A^{\prime}, \sigma\right) \in \mathrm{O}(m) \times \mathrm{O}(m) \times \mathrm{O}(2)
\end{gathered}
$$

Moreover, another look at the isometry classes of the $T$-orbits in $\left(M, \varphi g_{0}\right)$ shows that each $\Psi \in \mathcal{D}$ (see 2.1(ii)) will preserve the set $\left\{ \pm Z_{1}, \pm Z_{2}\right\} \subset \mathfrak{z}=\mathbb{R}^{2}$; in particular, $\Psi \in \mathrm{O}(2)$.

Proof of condition $(\mathrm{N})$.

Suppose condition $(\mathrm{N})$ were violated. Let $\Psi \in \mathcal{D}$ and $\bar{F} \in \overline{\operatorname{Aut}}_{\varphi g_{0}}^{T}(M)$ be such that $\Omega_{\lambda}=\Psi \circ \bar{F}^{*} \Omega_{\lambda^{\prime}}$. Note that we have $d \omega_{0}=0$ here and hence $\Omega_{0}=0$; thus $\Omega_{\lambda}=d \bar{\lambda}, \Omega_{\lambda^{\prime}}=d \bar{\lambda}^{\prime}$ with $\bar{\lambda}, \bar{\lambda}^{\prime}$ as in 2.1(iv). Let pr : $\mathbb{R}^{m} \oplus \mathbb{R}^{m} \oplus \mathbb{R}^{2} \rightarrow \mathbb{R}^{m} \oplus \mathbb{R}^{m}$ denote the canonical projection and also its restriction to $\hat{M} / T=B^{2 m+2} \cap Q$, resp. $\hat{M} / T=S^{2 m+1} \cap Q$. Then

$$
d \bar{\lambda}=\operatorname{pr}^{*}\left(d \nu \oplus d \nu^{\prime}\right), \quad d \bar{\lambda}^{\prime}=\operatorname{pr}^{*}\left(d \nu^{\prime} \oplus d \nu\right)
$$


For $X, Y \in T_{p} \mathbb{R}^{m}$ and $Z \in \mathfrak{z}$, we have

$$
\begin{aligned}
& \left\langle d \nu^{(\prime)}(X, Y), Z\right\rangle=2\left\langle j^{(\prime)}(Z) X, Y\right\rangle \quad(\text { in } 4.4), \text { resp. } \\
& \left\langle d \nu^{(\prime)}(X, Y), Z\right\rangle=3\left\langle c^{(\prime)}(Z) p \times X, Y\right\rangle, \quad(\text { in } 4.6)
\end{aligned}
$$

(compare the proof of [Sch3], 4.3, for the second equation). By (1), $\bar{F}$ is the restriction to $\hat{M} / T$ of some map $\left(A, A^{\prime}, \sigma\right) \in \mathrm{O}(m) \times \mathrm{O}(m) \times \mathrm{O}(2)$. Moreover, the interior of $\operatorname{pr}(\hat{M} / T) \in \mathbb{R}^{2 m}$ is the whole open unit ball $B^{2 m}$, regardless of whether $M=B$ or $M=S$. Therefore, one elementarily derives from the equation $d \bar{\lambda}=\Psi \circ \bar{F}^{*} d \bar{\lambda}^{\prime}$ and the formulas above:

$$
\begin{aligned}
& j^{\prime}\left({ }^{T} \Psi(Z)\right)=A j(Z) A^{-1}, \quad j\left({ }^{T} \Psi(Z)\right)=A^{\prime} j^{\prime}(Z) A^{\prime-1}, \quad \text { resp. } \\
& c^{\prime}\left(\operatorname{det}(A) \cdot{ }^{T} \Psi(Z)\right)=A c(Z) A^{-1}, \quad c\left(\operatorname{det}\left(A^{\prime}\right) \cdot{ }^{T} \Psi(Z)\right)=A^{\prime} c^{\prime}(Z) A^{\prime-1}
\end{aligned}
$$

for all $Z \in \mathfrak{z}$, where ${ }^{T} \Psi$ denotes the transpose of $\Psi$ with respect to the standard inner product on $\mathfrak{z}=\mathbb{R}^{2}$. Since $\Psi \in \mathrm{O}(\mathfrak{z})$ (see above) and $\operatorname{det}(A), \operatorname{det}\left(A^{\prime}\right) \in\{ \pm 1\}$, the equations in (2) contradict the nonequivalence assumption on $j, j^{\prime}$ from 4.5 , resp. on $c, c^{\prime}$ from 4.6 .

Proof of condition (G).

Suppose to the contrary that $\lambda^{\prime}$ did not have Property $(\mathrm{G})$; i.e., there is a nontrivial 1-parameter family $\bar{F}_{t} \in \overline{\operatorname{Aut}}_{\varphi g_{0}}^{T}(M)$ such that $\bar{F}_{t}^{*} \Omega_{\lambda^{\prime}} \equiv \Omega_{\lambda^{\prime}}$. Proceeding exactly as in the proof of condition $(\mathrm{N})$, replacing $\Psi$ by Id and $\lambda$ by $\lambda^{\prime}$, we now obtain 1-parameter families $A_{t}, A_{t}^{\prime} \in \mathrm{SO}(m)$ such that

$$
\begin{array}{ll}
j^{\prime}(Z) \equiv A_{t} j^{\prime}(Z) A_{t}^{-1}, & j(Z) \equiv A_{t}^{\prime} j(Z) A_{t}^{\prime-1}, \quad \text { resp. } \\
c^{\prime}(Z) \equiv A_{t} c^{\prime}(Z) A_{t}^{-1}, & c(Z) \equiv A_{t}^{\prime} c(Z) A_{t}^{\prime-1}
\end{array}
$$

for all $Z \in \mathfrak{z}$ and $t \in \mathbb{R}$ (compare (2)). The families $A_{t}, A_{t}^{\prime}$ are those occurring in $\bar{F}_{t}=\left(A_{t}, A_{t}^{\prime}, \mathrm{Id}\right) \in \mathrm{SO}(m) \times \mathrm{SO}(m) \times \mathrm{SO}(2)$. Note that the $\mathrm{SO}(2)$-component of $\bar{F}_{t}$ is trivial because $\bar{F}_{t}$ must preserve the open quadrant $Q$ of $\mathbb{R}^{2 m+2}$ considered earlier. Since the family $\bar{F}_{t}$ is assumed to be nontrivial, at least one of the 1-parameter families $A_{t}, A_{t}^{\prime}$ in $\mathrm{SO}(m)$ is nontrivial. The corresponding equations in (3) thus contradict the genericity assumptions from 4.5 on $j$ and $j^{\prime}$, resp. from 4.6 on $c$ and $c^{\prime}$.

\subsection{Nonisometry proof for Example 4.10.}

The proof will be similar to that of [Sch3], Proposition 4.3. Let $c, c^{\prime}: \mathfrak{z}=\mathbb{R}^{2} \rightarrow$ $\operatorname{Sym}_{0}\left(\mathbb{R}^{3}\right)$ be the pair of linear maps from Example 4.10 , and let $\nu, \nu^{\prime}$ be the as-

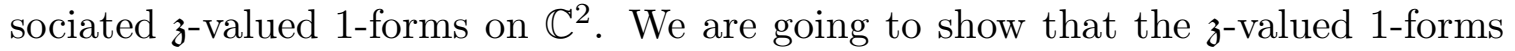
$\lambda, \lambda^{\prime}$ on $M=B^{8} \subset \mathbb{C}^{2} \oplus \mathbb{C}^{2}$ or $M=S^{7} \subset \mathbb{C}^{2} \oplus \mathbb{C}^{2}$, induced by $\nu \oplus \nu^{\prime}$, resp. $\nu^{\prime} \oplus \nu$, satisfy conditions $(\mathrm{N})$ and $(\mathrm{G})$ from Proposition 2.2 with respect to the metric $\varphi g_{0}$ 
on $M$, where $\varphi:=\varphi_{1}: \mathbb{C}^{2} \oplus \mathbb{C}^{2} \ni(p, q) \mapsto \psi(p) \in \mathbb{R}_{+}$. Since the metrics $\varphi_{1} g_{\lambda^{\prime}}$ and $\varphi_{2} g_{\lambda}$ on $M$ are isometric, as seen in the proof of Theorem 4.9, we may then conclude from Proposition 2.2 that the conformally equivalent metrics $\varphi_{1} g_{\lambda}$ and $\varphi_{2} g_{\lambda}$ are not isometric.

For $a, b \geq 0$ let $M_{a, b}:=\left\{\left.(p, q) \in \mathbb{C}^{2} \oplus \mathbb{C}^{2}|| p\right|^{2}=a^{2},|q|^{2}=b^{2}\right\}=S_{a}^{3} \times S_{b}^{3}$. Then $\hat{M}$ is the union of those $M_{a, b} \subset M$ with $a, b>0$ and $a^{2}+b^{2}<1$ (in case $M=B^{8}$ ), resp. $a^{2}+b^{2}=1$ (in case $M=S^{7}$ ).

Write $\psi(p)=\rho(|p|)$. The $T$-orbit of $(p, q) \in M_{a, b}$, endowed with the metric induced by $\varphi g_{0}$, is isometric to the rectangular torus with sides of length $\sqrt{\rho(a)} \cdot 2 \pi a$ and $\sqrt{\rho(a)} \cdot 2 \pi b$. Since $\rho$ is strictly increasing by assumption, so is the function $a \mapsto$ $\sqrt{\rho(a)} \cdot a$; note that this function maps $(0, \infty)$ bijectively to itself. Consequently, for every $(a, b) \in(0, \infty)^{2}$ there is exactly one $\left(a^{\prime}, b^{\prime}\right) \in(0, \infty)^{2}$ such that

$$
\sqrt{\rho\left(a^{\prime}\right)} \cdot a^{\prime}=\sqrt{\rho(a)} \cdot b \quad \text { and } \quad \sqrt{\rho\left(a^{\prime}\right)} \cdot b^{\prime}=\sqrt{\rho(a)} \cdot a
$$

(the first condition determines $a^{\prime}$, the second then determines $b^{\prime}$ ). The map $\sigma$ : $(a, b) \mapsto\left(a^{\prime}, b^{\prime}\right)$ on $(0, \infty)^{2}$ is continuous and obviously has the following property: Whenever $a>b$, then $a^{\prime}<a$ (since $a \mapsto \sqrt{\rho(a)} \cdot a$ is strictly increasing); hence $\sqrt{\rho\left(a^{\prime}\right)}<\sqrt{\rho(a)}$ and thus $\left\|\left(a^{\prime}, b^{\prime}\right)\right\|^{2}>\|(a, b)\|^{2}$. In particular, $\sigma(a, b)$ lies outside the closed unit disc for all $(a, b) \in \mathcal{C}:=\left\{(a, b) \in S^{1} \mid a>b>0\right\}$, and the same is true for some open neighborhood $\mathcal{A} \subset\{(a, b) \mid a>b>0\}$ of this $\operatorname{arc} \mathcal{C}$ of $S^{1}$. Choose $(a, b) \in \mathcal{C}$ (in case $\left.M=S^{7}\right)$, resp. $(a, b) \in \mathcal{U}:=\mathcal{A} \cap B_{1}(0)$ (in case $M=B^{8}$ ). Then $M_{a, b}$ is precisely the set of points in $\left(\hat{M}, \varphi g_{0}\right)$ whose $T$-orbit is isometric to the rectangular torus with sides of length $\sqrt{\rho(a)} \cdot 2 \pi a$ and $\sqrt{\rho(a)} \cdot 2 \pi b$.

Each $F \in \operatorname{Aut}_{\varphi g_{0}}^{T}(M)$ (see Definition 2.1) must therefore preserve $M_{a, b}=: L$. Note that $\left(L / T, \varphi g_{0}^{T}\right)$ is isometric to $\left(S_{a / 2}^{2} \times S_{b / 2}^{2}, \rho(a)^{2} g_{\text {stdd }}\right)$, where $g_{\text {stdd }}$ denotes the restriction of the standard metric on $\mathbb{R}^{3} \oplus \mathbb{R}^{3}$. Since $a>b$, every isometry of $\left(L / T, \varphi g_{0}^{T}\right)$ - and thus the restriction to $L / T$ of every $\bar{F} \in \overline{\operatorname{Aut}}_{\varphi g_{0}}^{T}(M)$ - must preserve the two factors. In particular, every $F \in \operatorname{Aut}_{\varphi g_{0}}^{T}(M)$ must preserve the factors of $M_{a, b}$; we conclude from this that every $\Psi \in \mathcal{D}$ is of the form $Z_{1} \mapsto \varepsilon_{1} Z_{1}$, $Z_{2} \mapsto \varepsilon_{2} Z_{2}$ with $\varepsilon_{1}, \varepsilon_{2} \in\{ \pm 1\}$; in particular, $\Psi \in \mathrm{O}(\mathfrak{z})$.

Proof of condition $(\mathrm{N})$.

Suppose condition (N) were violated. Let $\Psi \in \mathcal{D}$ and $\bar{F} \in \overline{\operatorname{Aut}}_{\varphi g_{0}}^{T}(M)$ be such that $\Omega_{\lambda}=\Psi \circ \bar{F}^{*} \Omega_{\lambda^{\prime}}$. Let $L=M_{a, b} \subset \hat{M}$ be chosen as above. By the arguments above, $\bar{F}$ induces an isometry of $\left(L / T, \varphi g_{0}^{T}\right)$. Denote by $\Omega_{\lambda}^{L}, \Omega_{\lambda^{\prime}}^{L}$ the $\mathbb{R}^{2}$-valued 2-forms induced by $\Omega_{\lambda}, \Omega_{\lambda^{\prime}}$ on $L / T \subset \hat{M} / T$; then $\Omega_{\lambda}^{L}=\Psi \circ \bar{F}^{*} \Omega_{\lambda^{\prime}}^{L}$ because $\bar{F}$ preserves $L / T$.

Observe that the first, resp. second, component of $\Omega_{0}^{L}$ is a scalar multiple of the standard volume form of the first, resp. second, factor of $L / T$ (compare [Sch3], 4.1(iii)). Recall from 2.1(iv) that $\Omega_{\lambda}=\Omega_{0}+d \bar{\lambda}, \Omega_{\lambda^{\prime}}=\Omega_{0}+d \bar{\lambda}^{\prime}$, and similarly for the 
forms induced on $L / T$. Note that each of $\Omega_{0}^{L}, d \bar{\lambda}^{L}$ and $d \bar{\lambda}^{\prime L}$ is a sum of pullbacks of forms defined on one of the factors of $L / T$. Since each 2-form on $S^{2}$ is uniquely decomposable into an exact component and a multiple of the volume form, and since $\bar{F}$ preserves the two factors of $L / T$, we therefore obtain $d \bar{\lambda}^{L}=\Psi \circ \bar{F}^{*} d \bar{\lambda}^{\prime L}$.

The restriction of $\bar{F}$ to $L / T$ is given as $\left(A, A^{\prime}\right)$ with $A, A^{\prime} \in \mathrm{O}(3)$. Evaluating the previous equation on 2-vectors tangent to the first factor of $L / T$, we get $d \bar{\nu}=$ $\Psi \circ A^{*} d \bar{\nu}^{\prime}$, where

$$
\left\langle d \bar{\nu}^{(\prime)}(X, Y), Z\right\rangle=3 a^{3}\left\langle c^{(\prime)}(Z) x \times X, Y\right\rangle
$$

for all $X, Y \in T_{x} S_{a / 2}^{2}$ and $Z \in \mathfrak{z}$; compare the proof of [Sch3], 4.3. As in [Sch3] one now derives

$$
c^{\prime}(\Phi(Z))=A c(Z) A^{-1}
$$

for all $Z \in \mathfrak{z}$, with $\Phi:=\operatorname{det} A \cdot \Psi \in \mathrm{O}(\mathfrak{z})$. But this contradicts the nonequivalence of $c$ and $c^{\prime}$ (see condition 2.) in 4.6).

Proof of condition (G).

Suppose to the contrary that $\lambda^{\prime}$ did not have Property $(\mathrm{G})$; i.e., there is a nontrivial 1-parameter family $\bar{F}_{t} \in \overline{\operatorname{Aut}}_{\varphi g_{0}}^{T}(M)$ such that $\bar{F}_{t}^{*} \Omega_{\lambda^{\prime}} \equiv \Omega_{\lambda^{\prime}}$. Note that $\bar{F}_{t}$, being an isometry of $\hat{M} / T$, is determined by its restriction to any nonempty open subset. The submanifolds $L / T=M_{a, b} / T$ do foliate an open subset of $\hat{M} / T$ if we vary the tuple $(a, b)$ in the set from which we had chosen it. It follows that $\bar{F}_{t}$ induces a nontrivial 1-parameter family of isometries on at least one of these $M_{a, b} / T$; again, we denote the corresponding $M_{a, b}$ by $L$.

Proceeding exactly as in the proof of condition $(\mathrm{N})$, replacing $\Psi$ by $\mathrm{Id}$ and $\lambda$ by $\lambda^{\prime}$, we now obtain 1-parameter families $A_{t}, A_{t}^{\prime}$ in $\mathrm{SO}(3)$, such that $d \bar{\lambda}^{\prime} L \equiv$ $\left(A_{t}, A_{t}^{\prime}\right)^{*} d \bar{\lambda}^{\prime L}$ on $L / T$. Evaluating this on the first, resp. second, factor of $L / T$, we see that $d \bar{\nu}^{\prime}$ is invariant under the $A_{t}$, resp. that $d \bar{\nu}$ is invariant under the $A_{t}^{\prime}$. We derive that

$$
c^{\prime}(Z) \equiv A_{t} c^{\prime}(Z) A_{t}^{-1} \quad \text { and } \quad c(Z) \equiv A_{t}^{\prime} c(Z) A_{t}^{\prime-1}
$$

for all $Z \in \mathfrak{z}$ (compare (4)). However, since at least one of the families $A_{t}, A_{t}^{\prime}$ is nontrivial, this contradicts the genericity assumption on $c$ and $c^{\prime}$ (see condition 3.) in 4.6).

\subsection{Nonisometry proof for the modified metrics from Remark 4.11.}

The case of Example 4.4/4.6.

We have to suitably adapt the proof given in 5.2. Note that $\lambda, \lambda^{\prime}$ did not play any role in the derivation of statement (1) of 5.2. In order to prove the conditions $(\mathrm{N})$ and $(\mathrm{G})$ for $f \lambda, f \lambda^{\prime}$, we proceed as follows: 
Choose any tuple $(r, s, t)$ such that $\alpha(r, s, t) \neq 0$; we can assume $r, s, t \neq 0$. Let $L:=\{(p, q, u) \in \hat{M}|| p|=r| q,|=s| u \mid,=t\}$. Then $f$ has the constant value $C:=\alpha(r, s, t) \neq 0$ on $L$. For all differential forms occurring in the proof of condition $(\mathrm{N})$ in 5.2 , we now restrict attention to the forms they induce on $L$, resp. on $L / T$. These induced forms are, up to multiplication by the constant $C$, the same as those induced by $f \lambda, f \lambda^{\prime}$ etc. The only problem in the course of the arguments from 5.2 is the fact that $\operatorname{pr}(L / T)=S_{r}^{m-1} \times S_{s}^{m-1}$ has empty interior in $\mathbb{R}^{2 m}$. But the equations in (2) can be derived nevertheless, even when using the equation $d \nu \oplus d \nu^{\prime}=\Psi \circ\left(A, A^{\prime}\right)^{*}\left(d \nu^{\prime} \oplus d \nu\right)$ only restricted to the tangent bundle of $S_{r}^{m-1} \times S_{s}^{m-1}$ :

In the case of Example 4.4, this is obvious from the formula for $d \nu^{(\prime)}$, because for all $X, Y \in \mathbb{R}^{m}$ there is certainly some $p \in S_{r}^{m-1}$ such that $X, Y \in T_{p} S_{r}^{m-1}$ (and similarly for $S_{s}^{m-1}$ ). In the case of Example 4.6, we first obtain only

$$
\left\langle\left(c(Z)-A^{-1} c^{\prime}\left(\operatorname{det}(A) \cdot{ }^{T} \Psi(Z)\right) A\right) p \times X, Y\right\rangle=0
$$

for all $Z \in \mathfrak{z}$ and all $X, Y \in T_{p} S_{r}^{2}$ (and similarly for $c, c^{\prime}$ reversed, with $A^{\prime}$ instead of $A$ ). But this implies $\left(c(Z)-A^{-1} c^{\prime}\left(\operatorname{det}(A) \cdot{ }^{T} \Psi(Z)\right) A\right) p \perp p$ for all $p \in S_{r}^{2}$. Since on the left hand side a symmetric map is applied to $p$, this map must be zero. Thus the equations in (2) follow nevertheless.

For both examples, the proof of condition $(G)$ now goes through verbatim.

The case of Example 4.10.

In case $M=B^{8}$, we make the assumption that the support of $\alpha$ nontrivially intersects $\mathcal{U}$, where $\mathcal{U} \subset B_{1}(0) \cap\{(a, b) \mid a>b>0\}$ is the nonempty open set occurring in the proof given in 5.3. In case $M=S^{7}$, since $\alpha$ does not identically vanish on the relevant parameter set $\left\{(a, b) \in S^{1} \mid a, b \geq 0\right\}$, and because of $\alpha(a, b)=\alpha(b, a)$, we know that $\alpha$ does not identically vanish on the $\operatorname{arc} \mathcal{C}=$ $\left\{(a, b) \in S^{1} \mid a>b>0\right\}$ either.

Thus we can choose $(a, b) \in \mathcal{U}$, resp. $(a, b) \in \mathcal{C}$, such that $C:=\alpha(a, b) \neq 0$. In 5.3 we saw that every $\bar{F} \in \overline{\operatorname{Aut}}_{\varphi g_{0}}^{T}(M)$ must then preserve $L:=M_{a, b}=S_{a}^{3} \times S_{b}^{3} \subset \hat{M}$. Moreover, $\left.f\right|_{L} \equiv C$. In order to prove the conditions $(\mathrm{N})$ and $(\mathrm{G})$ for $f \lambda, f \lambda^{\prime}$, we now work with $C \lambda^{L}, C \lambda^{\prime L}$ instead of $\lambda^{L}, \lambda^{\prime L}$ in 5.3, which does not affect any of the arguments.

\section{$\S 6$ OTHER TYPES OF EXAMPLES}

The techniques used in Sections 3 and 4 apply to the construction of isospectral conformally equivalent metrics and isospectral potentials in various settings. We first observe the common properties of the constructions, particularly the constructions of Section 3 and of Example 4.4. For simplicity, we will consider here linear maps $j$ from a vector space $\mathfrak{z}$ into $\mathfrak{s o}(m)$ rather than into a more general compact Lie algebra $\mathfrak{h}$. 
(i) Linear maps $j: \mathfrak{z} \rightarrow \mathfrak{s o}(m)$ where $\mathfrak{z}$ is a $k$-dimensional vector space, viewed as the Lie algebra of a torus $T$, were used to construct Riemannian metrics $g_{j}$ on a fixed manifold $M=M_{k, m}$; the manifold $M$ depended only on $k$ and $m$. (The metrics were denoted by $g_{\lambda^{j}}$ rather than $g_{j}$. In Section $3, M$ was a Lie group, while in Section $4, M$ was either a ball or sphere.) The torus $T$ acted by isometries on each $\left(M, g_{j}\right)$.

(ii) In each setting, we knew from previous work that $g_{j}$ and $g_{j^{\prime}}$ were isospectral whenever the linear maps $j$ and $j^{\prime}$ were isospectral in the sense of Definition 3.1. Moreover, the isospectrality of the metrics $g_{j}$ and $g_{j^{\prime}}$ had been proven using Theorem 1.3 with $\varphi=\psi=0$. Subtori $W \subset T$ of codimension one correspond to linear functionals $\mu \in \mathcal{L}^{*}$ or, equivalently (given an inner product on $\mathfrak{z}$ ) to elements $Z$ of $\mathfrak{z}$. The diffeomorphisms $F_{W}$ of Theorem 1.3 (referred to in Sections 3 and 4 as $F_{\mu}$ ) arose from the linear maps $A_{Z}$ in Defintion 3.1.

(iii) Given isospectral linear maps $j_{1}, j_{2}: \mathfrak{z} \rightarrow \mathfrak{s o}(m)$, we defined isospectral maps $j=\left(j_{1}, j_{2}\right)$ and $j^{\prime}=\left(j_{2}, j_{1}\right): \mathfrak{z} \rightarrow \mathfrak{s o}(2 m)$. The associated metrics $g_{j}$ and $g_{j^{\prime}}$ on $M=M_{k, 2 m}$ were isometric. Thus we had diffeomorphisms $F_{W}$ satisfying (ii), arising from the isospectrality of $j$ and $j^{\prime}$, and separately an isometry $\tau$ between $g_{j}$ and $g_{j^{\prime}}$. We then chose a conformal factor or potential $\varphi$ invariant under all the $F_{W}$ but not under $\tau$. The functions $\varphi$ and $\tau^{*} \varphi$ then gave isospectral potentials or conformal factors on $\left(M, g_{j}\right)$.

We now briefly describe how this procedure can be used to obtain isospectral potentials and conformal factors in other settings.

6.1 Example. Let $T$ be a torus with Lie algebra $\mathfrak{z}$. Let $N=N_{m}$ denote either the standard unit ball or the standard unit sphere in $\mathbb{R}^{m}$. Given $j: \mathfrak{z} \rightarrow \mathfrak{s o}(m)$, define

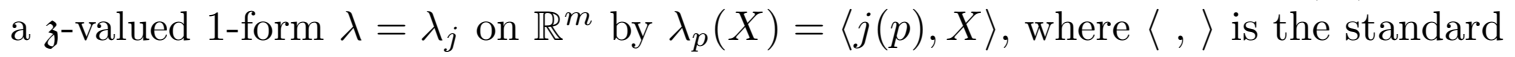
inner product on $\mathbb{R}^{m}$, and where $T_{p} \mathbb{R}^{m}$ is identified with $\mathbb{R}^{m}$. Then $\lambda$ pulls back, first by the inclusion, to a 1 -form on $N$ and then, by the canonical projection, to an admissible form on $N \times T$. Let $g_{j}$ denote the associated Riemannian metric on $N \times T$ as in Notation 1.4. As seen in [GW2] and [GGSWW], isospectral linear maps $j, j^{\prime}: \mathfrak{z} \rightarrow \mathfrak{s o}(m)$ result in isospectral metrics $g_{j}$ and $g_{j^{\prime}}$; in the notation of (ii) above, the role of the diffeomorphisms $F_{W}$ is played by the maps $A_{Z} \times$ Id : $N \times T \rightarrow N \times T$. In the notation of (iii), the role of $\tau$ is played by $\tau_{0} \times$ Id where $\tau_{0}$ is the diffeomorphism of the ball or sphere $N_{2 m}$ obtained by restriction of the map $(x, y) \mapsto(y, x)$ of $\mathbb{R}^{2 m}$. Choose a function $\psi$ on $N$ which is invariant under the action of $\mathrm{O}(m) \times \mathrm{O}(m)$ but which is not $\tau_{0}$-invariant. Pull $\psi$ back by the projection to $N \times T$ to obtain a function $\varphi$ satisfying the conditions of (iii).

6.2 Example. Let $T$ be a torus with Lie algebra $\mathfrak{z}$, and let $Q$ be a domain with boundary in the hyperbolic space $H^{m+1}$ invariant under the isometric action of $\mathrm{O}(m)$. With respect to the upper half space model of $H^{m+1}$ with coordinates $(a, x)$, $a \in \mathbb{R}^{+}, x \in \mathbb{R}^{m}$, the action of $\alpha \in \mathrm{O}(m)$ on $H^{m+1}$ is given by $\alpha(a, x)=(a, \alpha(x))$. 
The article [GSz] constructed from each linear map $j: \mathfrak{z} \rightarrow \mathfrak{s o}(m)$ a Riemannian metric $g_{j}$ on $Q \times T$. After multiplying the linear map $j$ by a small constant if necessary, the metric $g_{j}$ was shown to have negative curvature. Isospectral linear maps resulted in isospectral metrics, with the role of the diffeomorphisms in (ii) being played by the restriction to $Q \times T$ of the maps $A_{Z} \times \operatorname{Id}$ on $H^{m+1} \times T$. The procedure outlined in (iii) results in isospectral potentials or isospectral conformally equivalent metrics on $Q \times T$ where now $Q$ is an $\mathrm{O}(2 m)$-invariant domain in $H^{2 m+1}$. The role of $\tau$ in (iii) is played by the restriction to $Q \times T$ of $\tau_{0} \times \mathrm{Id}$, where $\tau_{0} \in \mathrm{O}(2 \mathrm{~m})$ is given as in Example 6.1. To obtain a function $\varphi$ as in (iii), begin with a function $\psi$ on $H^{2 m+1}$ invariant under the action of $\mathrm{O}(m) \times \mathrm{O}(m)$ but not $\tau_{0}$-invariant. The function $\varphi$ can then be taken to be the pullback to $Q \times T$ of $\left.\psi\right|_{Q}$. As in (iii), $\varphi$ and $\varphi \circ \tau$ give isospectral potentials on the negatively curved manifold $\left(Q \times T, g_{j}\right)$. By choosing $\varphi$ to be positive, one obtains isospectral conformally equivalent metrics on $Q \times T$. By moreover choosing $\varphi$ to be bounded by a sufficiently small constant, one can guarantee that the conformally equivalent metrics are negatively curved.

If we choose $m=4 \ell$ with $\ell \geq 2$, choose $\mathfrak{z}$ to be 3 -dimensional and choose $j_{1}, j_{2}: \mathfrak{z} \rightarrow \mathfrak{s o}(m)$ as in Theorem 3.8(i) of [GSz], then the metric $g_{j}$ on $Q \times T$, where $Q$ is a domain in $H^{2 m+1}$ and $j=\left(j_{1}, j_{2}\right)$, has constant Ricci curvature; indeed $\left(Q \times T, g_{j}\right)$ is a domain in a harmonic manifold. We thus obtain isospectral potentials on an Einstein manifold with boundary.

\section{REFERENCES}

[Ba] W. Ballmann, On the construction of isospectral manifolds, Preprint (2000).

[Be1] P. Bérard, Transplantation et isospectralité I, Math. Ann. 292 (1992), 547-559.

[Be2] Transplantation et isospectralité II, J. London Math. Soc. 48 (1993), 565-576.

[Br] R. Brooks, On manifolds of negative curvature with isospectral potentials, Topology 26 (1987), 63-66.

[BG] R. Brooks and C.S. Gordon, Isospectral families of conformally equivalent Riemannian metrics, Bull. Amer. Math. Soc. 23 (1990), no. 3, 433-436.

[BGG] R. Brooks, R. Gornet, and W. H. Gustafson, Mutually isospectral Riemann surfaces, Adv. Math. 138 (1998), 306-322.

[BPY] R. Brooks, P. Perry, and P. Yang, Isospectral sets of conformally equivalent metrics, Duke Math J. 58 (1989), 131-150.

[BT] R. Brooks and R. Tse, Isospectral surfaces of small genus, Nagoya Math. J. 107 (1987), 13-24.

[Bu] P. Buser, Isospectral Riemann surfaces, Ann. Inst. Fourier 36 (1986), 167-192.

[DG] D. DeTurck and C. Gordon, Isospectral Deformations II: trace formulas, metrics, and potentials, Comm. Pure Appl. Math. 42 (1989), 1067-1095.

[GGKM] C.S. Gardner, J.M. Greene, M.D. Kruskal, and R.M. Miura, A method for solving the Korteveg de Vries equation, Phys. Rev. Letters 19 (1967), 1095-1097.

[Gi] P. Gilkey, Recursion relations and the asymptotic behavior of the eigenvalues of the Laplacian, Compositio Math. 38, 201-240.

[Go1] C.S. Gordon, Isospectral closed Riemannian manifolds which are not locally isometric: II, Geometry of the Spectrum (R. Brooks, C. Gordon, P. Perry, eds.), Contemp. Math. 173 (1994), AMS, 121-131. 
[Go2] _ Survey of isospectral manifolds, Handbook of Differential Geometry, F.J.E. Dillen and L.C.A. Verstraelen, eds., vol. 1, Elsevier, 2000, pp. 747-778.

[Go3] _ Isospectral deformations of metrics on spheres, Invent. Math. 145 (2001), no. 2, 317-331.

[GGSWW] C. Gordon, R. Gornet, D. Schueth, D. Webb, E. Wilson, Isospectral deformations of closed Riemannian manifolds with different scalar curvature, Ann. Inst. Fourier 48 (1998), no. 2, 593-607.

[GSz] C.S. Gordon and Z.I. Szabó, Isospectral deformations of negatively curved Riemannian manifolds with boundary which are not locally isometric, Duke Math. J. 113 (2002), no. 2, 355-383.

[GWW] C. Gordon, D. Webb, and S. Wolpert, Isospectral plane domains and surfaces via Riemannian orbifolds, Invent. Math. 110 (1992), 1-22.

[GW1] C. Gordon and E.N. Wilson, Isospectral deformations of compact solvmanifolds, J. Diff. Geom. 19 (1984), 241-256.

[GW2] _ Continuous families of isospectral Riemannian metrics which are not locally isometric, J. Diff. Geom. 47 (1997), 504-529.

[Gt1] R. Gornet, A new construction of isospectral Riemannian nilmanifolds with examples, Michigan Math. J. 43 (1996), no. 1, 159-188.

[Gt2] Continuous families of Riemannian manifolds isospectral on functions but not on 1-forms, J. Geom. Anal. 10 (2000), no. 2, 281-298.

[Ik1] A. Ikeda, Isospectral problem for spherical space forms, Spectra of Riemannian Manifolds (M. Berger, S. Murakami, and T. Ochiai, eds.), Kaigai Publications, 1983, pp. 57-63.

[Ik2] 는 Riemannian manifolds $p$-isospectral but not $(p+1)$-isospectral, Geometry of Manifolds (Matsumoto), Perspect. Math., vol. 8, Academic Press, Boston, MA, 1989, pp. 383-417.

[MR1] R. Miatello and J.P. Rossetti, Flat manifolds isospectral on p-forms, J. Geom. Anal. 11 (2001), 649-667.

[MR2] _ Comparison of twisted p-form spectra for flat manifolds with diagonal holonomy, to appear, Ann. Global Anal. Geom.

[MR3] Length spectra and p-spectra of compact flat manifolds, preprint.

[Mi] J. Milnor, Eigenvalues of the Laplace operator on certain manifolds, Proc. Nat. Acad. Sci. USA 51 (1964), 542.

[OT] T. Ochiai and T. Takahashi, The group of isometries of a left invariant Riemannian metric on a Lie group, Math. Ann. 223 (1976), 91-96.

[Pe1] H. Pesce, Représentations relativement équivalentes et variétés Riemanniennes isospectrales, C. R. Acad. Sci. Paris, Série I 3118 (1994), 657-659.

[Pe2] _ Quelques applications de la théorie des représentations en géométrie spectrale, Rend. Mat., Série VII 18 (1998), 1-63.

[PT] J. Pöschel and E. Trubowitz, Inverse spectral theory, Pure and Applied Mathematics, vol. 130, Academic Press, 1987.

[Sch1] D. Schueth, Continuous families of isospectral metrics on simply connected manifolds, Ann. of Math. (2) 149 (1999), no. 1, 287-308.

[Sch2] Isospectral manifolds with different local geometries, J. Reine Angew. Math. $\mathbf{5 3 4}$ (2001), 41-94.

[Sch3] _ Isospectral metrics on five-dimensional spheres, J. Diff. Geometry 58 (2001), no. 1, 87-111.

[Sp] M. Spivak, Differential Geometry III, Publish or Perish, 1975.

[Su] T. Sunada, Riemannian coverings and isospectral manifolds, Ann. of Math. (2) 121 (1985), 169-186. 
[St] C. Sutton, Isospectral simply-connected homogeneous spaces and the spectral rigidity of group actions, to appear, Comment. Math. Helv.

[Sz1] Z.I. Szabó, Locally non-isometric yet super isospectral spaces, Geom. Funct. Anal. 9 (1999), no. 1, 185-214.

[Sz2] Isospectral pairs of metrics constructed on balls, spheres, and other manifolds with different local geometries, Ann. of Math. (2) 154 (2001), no. 2, 437-475.

[Sz3] Cornucopia of isospectral pairs of metrics on balls and spheres with different local geometries, Preprint (2000), math.DG/0011034.

[Ur] H. Urakawa, Bounded domains which are isospectral but not congruent, Ann. Scient. Éc. Norm. Sup. 15 (1982), 441-456.

[Vi] M.-F. Vignéras, Variétés Riemanniennes isospectrales et non isométriques, Ann. of Math. 112 (1980), 21-32.

Dartmouth College, Hanover, New Hampshire 03755, U.S.A.

E-mail address: csgordon@dartmouth.edu

Mathematisches Institut, Universität Bonn, Beringstr. 1, D-53115 Bonn, Germany

E-mail address: schueth@math.uni-bonn.de 